\title{
Should the Exchange Rate be in the Monetary Policy Objective Function?*
}

\author{
Tatiana Kirsonova ${ }^{\dagger}$ \\ University of Exeter
}

\author{
Campbell Leith ${ }^{\ddagger}$ \\ Campbell Leith $\ddagger$
Glasgow University
}

November 1, 2004

\author{
Simon Wren-Lewis ${ }^{\S}$ \\ University of Exeter
}

\begin{abstract}
Car 1,2004
Abstract

Following from Woodford's derivation of a benevolent monetary policy maker's objective function from agents utility, a number of papers have suggested that policy in an open economy should have the same objectives as in a closed economy, and in particular that the exchange rate should play no role. We show that this conclusion is not robust to the presence of any shocks to International Risk Sharing. When such shocks are important, the exchange rate appears alongside output and inflation in the social welfare function. However the form of this target is rather different from that used by a number of authors.
\end{abstract}

\footnotetext{
* Our thanks to Mike Artis, Chris Allsopp, David Cobham, and particularly Alan Sutherland.

${ }^{\dagger}$ Address: University of Exeter, School of Business and Economics, Streatham Court, Rennes Drive Exeter EX4 4PU; e-mail: t.kirsanova@exeter.ac.uk

${ }^{\ddagger}$ Address: University of Glasgow, Department of Economics, Glasgow G12 8RT; e-mail: c.b.leith@socsci.gla.ac.uk

$\S$ Address: University of Exeter, School of Business and Economics, Streatham Court, Rennes Drive Exeter EX4 4PU; e-mail: s.wren-lewis@exeter.ac.uk
} 


\section{Introduction}

Woodford (2003), following Rotemberg and Woodford (1997), showed that by taking a second order expansion of a representative agent's utility it was possible to derive a benevolent monetary policy maker's objective function, which took the traditional form involving quadratic terms in inflation and the output gap. This microfounding of a social welfare function has been rapidly adopted in the literature. Some recent papers have extended the analysis to an open economy, and reached the conclusion that the form of the social welfare function remains unchanged. For example, Aoki (2002) considers a two sector model, where prices in one sector are completely flexible, and shows that it is only inflation in the non-flexible sector that is relevant for welfare. He explicitly suggests that imported goods in an open economy are akin to the flexible price sector, and that therefore the price of imported goods (and by implication the exchange rate) should not appear in the inflation measure representing welfare. Gali and Monacelli (2002) consider a small open economy and come to the same conclusion, although the result is only demonstrated in the special case where utility functions are logarithmic. Clarida, Gali, and Gertler (2001) use the same model, and suggest that this result holds for any constant elasticity formulation of the utility functions. In all cases the exchange rate does not enter the benevolent policy maker's objective function.

In this paper we show that this result will no longer hold once we allow for one important source of distortionary shocks, which are deviations away from international risk sharing or uncovered interest parity. Once we allow for these shocks, inflation and the output gap are no longer sufficient to summarise aggregate welfare, and there is a role for a terms of trade or exchange rate term as well. While some authors have experimented with including the exchange rate alongside inflation and output as monetary policy targets (e.g. Kollmann (2002)), as far as we are aware none have used the exchange rate in the form suggested by our welfare analysis.

The structure of the paper is as follows. In Section 2 we outline the model we use. We show how international risk sharing or uncovered interest parity shocks mean that the output gap and consumption gap can move in different directions. In Section 3 we derive a second order expansion of the model's represent agents utility function, and show how this introduces a role for the exchange rate gap alongside the output gap in the social welfare function. Section 4 summarises the analysis. In the main text we try and focus on the economics behind our analysis, and full algebraic derivations are given in an appendix.

\section{Deriving the Model}

Our model is of a small open economy, and represents a simple extension to the model outlined in Gali and Monacelli (2002) and used in Clarida, Gali, and Gertler (2001). The model involves 
the following key elements. There is no capital or government expenditure. There are two types of consumption good; those produced domestically and overseas. The law of one price holds for any particular good. Firms are monopolistically competitive, which on its own would mean that aggregate output is permanently below efficient levels, but the government can set a subsidy to eliminate this distortion in steady state. Prices are set according to Calvo contracts, which generates an aggregate externality that is responsible for business cycles. The benevolent monetary policy maker sets interest rates to counteract this distortion. Following the literature, we describe the "natural' level of variables as the level that would occur in the absence of nominal inertia.

Our focus in this section is to derive equations in terms of first order log linear deviations from steady state. (In deriving our expression for welfare, we will in fact need second order expansions for some of these equations, but their derivation is given in an appendix.) We will examine the conditions under which the output gap, that is deviations in output from its natural level, fully describes similar gaps for other variables. This question is crucial for assessing whether the exchange rate has an independent role in influencing social welfare.

\subsection{Home and Foreign goods}

The representative household maximises

$$
\max _{\left\{C_{s}, y_{s}\right\}_{s=t}^{\infty}} E_{t} \sum_{s=t}^{\infty} \beta^{s-t}\left[u\left(C_{s}, \xi_{s}\right)-v\left(y_{s}(z), \xi_{s}\right)\right]
$$

where $C$ is consumption, $y$ is output for good $z, \xi$ is a taste shock, and $\beta$ is a discount rate. We generalise Clarida, Gali, and Gertler (2001) by including preference shocks and not specifying the functional form of the utility function ${ }^{1}$. The advantage of the former is that it allows us to focus more precisely on when exchange rate movements may have implications for welfare. The advantage of the latter is that it strongly motivates deriving second order approximations to welfare. (The function $v(\cdot)$ embodies both the disutility of labour and the production technology.). The aggregate consumption bundle is given by

$$
C=\left[(1-\alpha)^{\frac{1}{\eta}} C_{H}^{\frac{\eta-1}{\eta}}+\alpha^{\frac{1}{\eta}} C_{F}^{\frac{\eta-1}{\eta}}\right]^{\frac{\eta}{\eta-1}}
$$

where $C_{H}$ and $C_{F}$ are indices of consumption of domestic and foreign goods, and we drop the time subscript wherever all variables are dated at $t$. The parameter $\alpha$ is related to the share of imported goods in domestic consumption. In turn

\footnotetext{
${ }^{1}$ We assume CES utility in several derivations to illustrate some points, but without loss of generality.
} 


$$
C_{H}=\left[\int_{0}^{1} c_{H}^{\frac{\epsilon-1}{\epsilon}}(z) d z\right]^{\frac{\epsilon}{\epsilon-1}}, \quad C_{F}=\left[\int_{0}^{1} c_{F}^{\frac{\epsilon-1}{\epsilon}}(z) d z\right]^{\frac{\epsilon}{\epsilon-1}}, \quad \epsilon>1
$$

The optimal allocation of any given expenditure within each category of goods yields the demand functions

$$
c_{H}(z)=\left(\frac{p_{H}(z)}{P_{H}}\right)^{-\epsilon} C_{H}, \quad c_{F}(z)=\left(\frac{p_{F}(z)}{P_{F}}\right)^{-\epsilon} C_{F}, \quad z \in[0,1]
$$

where

$$
P_{H}=\left[\int_{0}^{1} p_{H}^{1-\epsilon}(z) d z\right]^{\frac{1}{1-\epsilon}}, \quad P_{F}=\left[\int_{0}^{1} p_{F}^{1-\epsilon}(z) d z\right]^{\frac{1}{1-\epsilon}}
$$

The optimal allocation of expenditures between domestic and foreign goods implies, given the law of one price

$$
C_{H}=(1-\alpha)\left(\frac{P_{H}}{P}\right)^{-\eta} C, \quad C_{F}=\alpha\left(\frac{P_{F}}{P}\right)^{-\eta} C
$$

where the consumer price index $(\mathrm{CPI})$ is

$$
P=\left((1-\alpha) P_{H}^{1-\eta}+\alpha P_{F}^{1-\eta}\right)^{\frac{1}{1-\eta}}
$$

We can also define the home output index $Y$ as

$$
Y=\left[\int_{0}^{1} y(z)^{\frac{\epsilon-1}{\epsilon}} d z\right]^{\frac{\epsilon}{\epsilon-1}}
$$

\subsection{Terms of trade and real exchange rate}

We assume that the law of one price holds

$$
\epsilon=\frac{P_{F}}{P_{F}^{*}}
$$

We define the terms of trade as

$$
S=\frac{P_{F}}{P_{H}}=\frac{P_{F}^{*}}{P_{H}^{*}}
$$

and the real exchange rate

$$
Q=\frac{\epsilon P^{*}}{P}
$$

We also assume a symmetric equilibrium and that the second economy is large, so that

$$
\begin{aligned}
P_{F}^{*} & =P^{*} \\
Y^{*} & =C^{*}
\end{aligned}
$$




\subsection{Intertemporal optimisation}

Each household faces the same flow budget constraint:

$$
P_{t} C_{t}+E_{t}\left[R_{t, t+1} B_{t+1}\right] \leq B_{t}+W_{t} N_{t}+T_{t}
$$

where $B_{t+1}$ is the nominal payoff in period $t+1$ of a portfolio held at the end of period $t, R$ is a stochastic discount factor for nominal payoffs, $W$ is the nominal wage, $N$ labour supply and $T$ denotes lump sum transfers/taxes. The riskless short term interest rate, $i_{t}$, is given by

$$
\frac{1}{1+i_{t}}=E_{t}\left(R_{t, t+1}\right)
$$

Together with transversality conditions, the budget constraint can be solved forward to yield:

$$
\sum_{s=t}^{\infty} E_{t}\left(R_{t, s} P_{s} C_{s}\right) \leq B_{t}+\sum_{s=t}^{\infty} E_{t}\left(R_{t, s}\left[W_{s} N_{s}+T_{s}\right]\right)
$$

Assume a linear production technology, such that $N_{t}=y_{t}(z)$. The Lagrangian and first order conditions are

$$
\begin{aligned}
L & =E_{t} \sum_{s=t}^{\infty} \beta^{s-t}\left[u\left(C_{s}, \xi_{s}\right)-v\left(y_{s}(z), \xi_{s}\right)\right] \\
& -\lambda\left[\sum_{s=t}^{\infty} E_{t}\left(R_{t, s} P_{s} C_{s}\right)-B_{t}-\sum_{s=t}^{\infty} E_{t}\left(R_{t, s}\left[W_{s} y(z)_{s}+T_{s}\right]\right)\right. \\
\frac{\partial L}{\partial y_{s}(z)} & =-\beta^{s-t} v_{h}\left(y_{s}, \xi_{s}\right)+\lambda R_{t, s} W_{s}=0 \\
\frac{\partial L}{\partial C_{s}} & =\beta^{s-t} u_{C}\left(C_{s}, \xi_{s}\right)-\lambda R_{t, s} P_{s}=0
\end{aligned}
$$

The consumption first order condition can be rewritten as:

$$
\beta \frac{u_{C}\left(C_{t+1}, \xi_{t+1}\right)}{u_{C}\left(C_{t}, \xi_{t}\right)} \frac{P_{t}}{P_{t+1}}=\frac{1}{1+i_{t}}
$$

and we can also write the leisure/consumption trade off

$$
\frac{v_{y}(y, \xi)}{u_{C}(C, \xi)}=\frac{W}{P}
$$

Log-linearising the definition of consumer prices around a steady state where $P_{H}=P_{F}$ gives

$$
\hat{P}=(1-\alpha) \hat{P}_{H}+\alpha \hat{P}_{F}=\hat{P}_{H}+\alpha \hat{S}
$$

where for any variable $x, \hat{x}$ denotes log-linear deviations from steady state. Denoting inflation as $\pi_{t+1}=\ln \left(P_{t+1} / P_{t}\right)$, then we can also write 


$$
\pi=\pi_{H}+\alpha \Delta \hat{S}
$$

where $\pi$ is CPI inflation, and $\pi_{H}$ output price inflation. Using the first order expansion

$$
U_{C}\left(C_{t}, \xi_{t}\right)=U_{C}\left(C_{t}, \xi_{t}\right)\left(1+\frac{U_{C C}(C, \xi) C \hat{C}_{t}}{U_{C}(C, \xi)}+\frac{U_{C \xi}(C, \xi) \hat{\xi}_{t}}{U_{C}(C, \xi)}\right)
$$

and a similar expression for $v_{y}$ allows us to derive

$$
\begin{aligned}
\hat{W}-\hat{P}_{H}-\alpha \hat{S} & =\left[\hat{y} \frac{1}{\psi}+\hat{C} \frac{1}{\sigma}+\hat{\xi}\left(\frac{h}{\psi}+\frac{g}{\sigma}\right)\right] \\
\hat{C}_{t} & =E_{t}\left[\hat{C}_{t+1}\right]-\sigma\left(r_{t}-E_{t}\left[\pi_{t+1}\right]\right)
\end{aligned}
$$

where

$$
\begin{aligned}
\sigma & =-\frac{U_{C}(\cdot)}{U_{C C}(\cdot) C} \\
g & =\frac{U_{C \xi}(\cdot)}{U_{C C}(\cdot) C}=-\frac{U_{C \xi}(\cdot) \sigma}{U_{C}(\cdot)} \\
\psi & =\frac{v_{y}(\cdot)}{v_{y y}(\cdot) y} \\
h & =\frac{v_{y \xi}(\cdot)}{v_{y y}(\cdot) y}=\frac{v_{y \xi}(\cdot) \psi}{v_{y}(\cdot)}
\end{aligned}
$$

The left hand side of $(22)$ is the real consumer wage. Note that with logarithmic utility, which is the case considered by Gali and Monacelli (2002), we have $\sigma=1, \psi=-1, g=h=0$.

To derive a log-linearised demand curve for home goods, note that these goods are either consumed at home or abroad, so that

$$
\begin{aligned}
& Y=C_{H}+C_{H}^{*} \\
& \hat{Y}=(1-\alpha) \hat{C}_{H}+\alpha \hat{C}_{H}^{*}
\end{aligned}
$$

to first order. From the demand curve we have

$$
\hat{C}_{H}-\hat{C}=-\eta\left(\hat{P}_{H}-\hat{P}\right)=\eta \alpha \hat{S}
$$

We can write a similar relationship for foreign consumers: 


$$
\hat{C}_{H}^{*}-\hat{C}^{*}=-\eta\left(\hat{P}_{H}^{*}-\hat{P}^{*}\right)
$$

As the rest of the world is large, $C^{*}=Y^{*}$ and $P^{*}=P_{F}$, so output is given by

$$
\begin{aligned}
\hat{Y} & =(1-\alpha)(\hat{C}+\eta \alpha \hat{S})+\alpha\left(\hat{Y}^{*}+\eta \hat{S}\right) \\
& =\alpha \hat{Y}^{*}+(1-\alpha) \hat{C}+\alpha \eta(2-\alpha) \hat{S}
\end{aligned}
$$

where we ignore second order or higher terms. Thus the demand for domestic output depends on domestic consumption, world output and the real exchange rate.

\subsection{Price setting}

Price setting follows the usual Calvo set-up with $1-\gamma$ of firms changing price in a given period. The log-linear pricing rule for prices changed in period $t$ is given by,

$$
\tilde{p}_{H, t}=\mu+(1-\beta \gamma) \sum_{k=0}^{\infty}(\beta \gamma)^{k} E_{t}\left\{m c_{t+k}\right\}
$$

where $\mu=\log \left(\frac{\varepsilon}{\varepsilon-1}\right)$ is the gross mark-up in the steady-state, and $m c$ are nominal marginal costs. The following log-linear approximation

$$
\pi_{H, t}=(1-\gamma)\left(\tilde{p}_{H, t}-p_{H, t-1}\right)
$$

allows us to write (set $p_{H, t-1}=p_{H, t}-\pi_{H, t}$ to obtain an equation for $\gamma \pi_{H, t}$ and subtract $\beta \gamma \pi_{H, t+1}$ )

$$
\pi_{H, t}=\beta E_{t}\left[\pi_{H, t+1}\right]+\frac{(1-\gamma)(1-\beta \gamma)}{\gamma} \widehat{m c}_{t}
$$

This is a Phillips curve of the 'New Keynesian' type. Real marginal costs $\widehat{m c}$ are given by

$$
\widehat{m c}=\hat{W}-\hat{P}_{H}
$$

and, finally, the linear Phillips curve can be written as (see Appendix A.3, formula (79)):

$$
\pi_{H, t}=\beta \pi_{H, t+1}+\frac{\psi(1-\gamma \beta)(1-\gamma)}{(\epsilon+\psi) \gamma}\left(\frac{1}{\psi} \hat{Y}_{t}+\frac{1}{\sigma} \hat{C}_{t}+\alpha \hat{S}_{t}-\left(\frac{1}{\sigma}+\frac{1}{\psi}\right) \hat{\xi}_{t}\right) .
$$




\subsection{International risk sharing}

With complete securities markets, the Euler equation

$$
\beta \frac{u_{C}\left(C_{t+1}^{i}, \xi_{t+1}\right)}{u_{C}\left(C_{t}^{i}, \xi_{t}\right)} \frac{P_{t}}{P_{t+1}}=R_{t, t+1}
$$

must also hold for the rest of the world, i.e.

$$
\beta \frac{u_{C}\left(C_{t+1}^{*}, \xi_{t+1}\right)}{u_{C}\left(C_{t}^{*}, \xi_{t}\right)} \frac{P_{t}^{*}}{P_{t+1}^{*}}=R_{t, t+1}^{*}
$$

Perfect arbitrage would suggest

$$
E_{t}\left[\frac{R_{t, t+1}^{*} \epsilon_{t+1}}{\epsilon_{t}}\right]=E_{t}\left[R_{t, t+1}\right]
$$

which is Uncovered Interest Parity when $\frac{1}{1+i_{t}}=E\left[R_{t, t+1}\right]$ and $\frac{1}{1+i_{t}^{*}}=E\left[R_{t, t+1}^{*}\right]$. We want to introduce a distortion, such that

$$
\frac{\epsilon_{t} \zeta_{t}}{E_{t}\left[\epsilon_{t+1} \zeta_{t+1}\right]\left(1+i_{t}^{*}\right)}=\frac{1}{1+i_{t}}
$$

where $\zeta_{t}$ is the distortionary shock. The reason for adding such shocks is straightforward. One argument that is frequently invoked in favour of exchange rate targeting (and its limit, monetary union) is that markets often drive the exchange rate well away from levels implied by fundamentals, and that this has damaging effects on the economy as a whole. (For example, see Buiter and Grafe (2003) in evidence submitted to the U.K. Treasury enquiry into joining EMU.) The empirical importance of distortions to uncovered interest parity are discussed in Kollmann (2003) and Kollmann (2002). It is therefore important to investigate whether these shocks introduce a role for the exchange rate in the welfare function, and what form that might take. Using this relationship with the two Euler equations gives

$$
\frac{u_{C}\left(C_{t+1}, \xi_{t+1}\right) u_{C}\left(C_{t}^{*}, \xi_{t}^{*}\right)}{u_{C}\left(C_{t+1}^{*}, \xi_{t+1}^{*}\right) u_{C}\left(C_{t}, \xi_{t}\right)} \frac{\zeta_{t}}{\zeta_{t+1}}=\frac{Q_{t}}{Q_{t+1}}
$$

where $Q$ is the real exchange rate defined above. By integrating we can show that home consumption will be related to world consumption, the real exchange rate, and the international risk sharing (IRS) distortion. A first order linearised version, which assumes that $\xi$ and $\xi^{*}$ are identical $^{2}$, is given by

$$
\hat{C}_{t}=\hat{Y}_{t}^{*}+\sigma(1-\alpha) \hat{S}_{t}-\sigma \hat{\zeta}_{t}+O(2)
$$

\footnotetext{
${ }^{2}$ Departures from this assumption would add terms similar to $\zeta$, but a key distinction is that $\zeta$ is distortionary while $\xi$ reflects preferences.
} 


\subsection{Gap variables}

Equations (42) and (32) represent a two equation system involving three endogenous variables (output, consumption and the terms of trade), one exogenous variable (world output) and the IRS shock. The latter is a distortion, and so it is appropriate to define the natural level of a variable as the level that would occur in the absence of this distortion as well as nominal inertia. We can therefore derive the following two equations in terms of 'gaps' i.e. the difference between actual and natural deviations from steady state.

$$
\begin{aligned}
& \hat{Y}_{t}-\hat{Y}_{t}^{n}=(1-\alpha)\left(\hat{C}_{t}-\hat{C}_{t}^{n}\right)+\alpha \eta(2-\alpha)\left(\hat{S}_{t}-\hat{S}_{t}^{n}\right) \\
& \hat{C}_{t}-\hat{C}_{t}^{n}=\sigma(1-\alpha)\left(\hat{S}_{t}-\hat{S}_{t}^{n}\right)-\sigma \hat{\zeta}_{t}
\end{aligned}
$$

where the superscript ${ }^{n}$ denotes natural levels, and we ignore terms of order two or more.

If $\hat{\zeta}_{t}=0$, as in Clarida, Gali, and Gertler (2001), then these two equations allow us to express both the consumption and terms of trade gaps as proportionate to the output gap to first order. We only need to know what the output gap is to know exactly what the other two gaps will be, so if these other gaps appear in any expression for social welfare, we can always replace them by terms in the output gap. However, once we allow for an international risk sharing distortion, this will no longer be the case. Such a shock may generate a terms of trade or consumption gap, yet the output gap could be zero. As we shall now show, this is crucial in establishing a potential role for the exchange rate in a social welfare function.

\section{Social Welfare with IRS shocks}

Following Woodford's analysis (Woodford (2003)), there are now a large number of papers that derive policy makers objective functions explicitly from consumers' utility (besides those cited above, other examples are Sutherland (2002a), Batini, Harrison, and Millard (2003), De Paoli (2004) and Pappa (2002)). Rather than assume and evaluate some specific and arbitrary intraperiod utility function, the analysis aims to present a second-order approximation of a general function, which would include only known parameters, including some shape characteristics like risk-aversion. In the analysis below we try and focus on the economics behind this derivation, leaving the detailed maths to an appendix.

A benevolent policy maker will attempt to maximise the utility of all workers, and so will maximise the discounted sum of terms which at each time $s$ will be of the form

$$
U\left(C_{s}, \xi_{s}\right)-\int_{0}^{1} v\left(y_{s}(z), \xi_{s}\right) d z
$$

Taking a second order approximation of (43) around the steady state, and dropping the common time subscript for clarity, gives 


$$
\begin{aligned}
\mathcal{W} & =U(C, \xi)-\int_{0}^{1} v(y(z), \xi) d z \\
& =U_{c}(\cdot) C\left[\hat{C}\left(1-\frac{g}{\sigma} \hat{\xi}\right)+\frac{1}{2}\left(1-\frac{1}{\sigma}\right) \hat{C}^{2}\right] \\
& -Y v_{y}(\cdot)\left[\hat{Y}\left(1+\frac{h}{\varphi} \hat{\xi}\right)+\frac{1}{2}\left(1+\frac{1}{\psi}\right) \hat{Y}^{2}+V_{z} \hat{y}(z) \frac{1}{2}\left(\frac{1}{\psi}+\frac{1}{\epsilon}\right)\right]+t i p(3)
\end{aligned}
$$

where $V_{z}$ denotes the variance across goods, and $\operatorname{tip}(3)$ represents terms that are independent of policy (i.e. terms involving the steady state or shocks alone) and terms higher than second order (see Appendix B). The first two terms come from the utility of consumption, and represent the costs of aggregate consumption deviating from its optimal path. The next two terms represent similar magnitudes for labour supply and hence output, while the final term represents costs associated with the output of individual goods differing from average output.

As Woodford (2003) shows, the term in the variance of output across producers exists because of the distortion due to Calvo contracts and can be replaced with a quadratic term in inflation. (The derivation for our model is exactly the same, so there is little point in repeating it here.) With nominal inertia in the form of Calvo contracts, the only reason for output of individual goods to differ is that some firms change their prices while others do not. The greater is inflation, the larger the movement in relative prices, and therefore the larger the variance in output across goods. This is a particularly clear representation of one of the standard arguments for costs associated with inflation: that higher inflation is associated with a greater distortion in relative prices, and therefore a larger misallocation of production and labour across goods. However it also apparent from this derivation that it is only the relative price of domestically produced goods, and therefore output price inflation, that is relevant for welfare, because only domestic goods are involved in domestic labour supply. To put this another way, inflation only matters to agents as workers, not as consumers. The exchange rate therefore has no 'direct' role in this inflation measure.

What about the remaining terms? These involve either output or consumption (and the preference shock), so in this very basic sense there is no role for the exchange rate or the terms of trade. However the welfare function in this form is not very useful to policy makers for two reasons. First, it involves terms in the preference shock, which may not be known. Second, it is unclear what relationship this expression has to more conventional linear quadratic objective functions. Both problems can potentially be resolved by utilising the results of individual agents optimising behaviour. In doing this, we want to focus on two key issues. First, can the terms in consumption be replaced by terms in output alone, or will we also require terms in the terms of trade? Second, does the analysis imply that the policy maker should attempt to achieve the 
flex-price equilibrium, which would be the case if welfare could be written as quadratic terms in gaps alone.

These issues can be examined most transparently if we split the welfare expression into four groups, where the fourth involves the terms in the variance across goods already discussed. The first group, which we denote by $\mathcal{W}_{L}$, involves only linear terms in consumption and output: ceteris paribus more consumption is good, and more labour supply is bad:

$$
\mathcal{W}_{L}=U_{c} C \hat{C}-Y v_{y} \hat{Y}
$$

An obvious question is whether, by using the work/leisure trade-off involved in agents optimisation, we can eliminate these terms? In a closed economy, $C=Y$ and $\hat{C}=\hat{Y}$, but in general it will not be the case that $U_{c}=v_{y}$, because of the monopolistic distortion. Rotemberg and Woodford (1997) sidestepped this problem by assuming a production subsidy that exactly offset the impact of monopoly production, ensuring that $\mathcal{W}_{L}=0$. In the main text we follow this route for our open economy. More recently Sutherland (2002b) and Benigno and Woodford (2004) have used an alternative procedure, which involves using a second order approximation of the Phillips curve. This approach is only valid under particular policies ${ }^{3}$. We consider this approach in the Appendix, and find that our key results also apply in this case.

In an open economy, we can use (32) and (42) to write

$$
\hat{C}=\Phi \hat{Y}+\hat{X}
$$

where

$$
\begin{aligned}
\hat{X} & =\frac{1}{2} \Phi \hat{Y}^{2}-\Phi \alpha^{2} \eta(2-\alpha) \hat{S}^{2}-\frac{1}{2} \frac{\Phi}{\Phi_{b}} \hat{C}^{2}-\Phi \alpha \eta \hat{S} \hat{Y}^{*} \\
& -\Phi \frac{\alpha \eta}{\sigma}((2-\alpha)+\sigma(1-\alpha)) \hat{S} \hat{C}+\operatorname{tip}(3)
\end{aligned}
$$

and $\Phi$ is a combination of model parameters (see Appendix B for details). $\hat{X}$ involves terms of order two and will not disappear even for logarithmic utility ${ }^{4}$. Note that although $\hat{X}$ involves only terms of order two, it does imply that in general the relationship between output and consumption will be influenced by changes in exogenous world demand or preference shocks. Substituting this into the expression for $\mathcal{W}_{L}$ implies

$$
\begin{aligned}
\mathcal{W}_{L} & =U_{c} C(\Phi \hat{Y}+\hat{X})-Y v_{y} \hat{Y}+\operatorname{tip}(3) \\
& =\hat{Y}\left(U_{c} C \Phi-Y v_{y}\right)+U_{c} C \hat{X}+\operatorname{tip}(3)
\end{aligned}
$$

\footnotetext{
${ }^{3}$ Sutherland (2002b) considers some particular rules for the money supply and Benigno and Woodford (2004) derive results for the timeless-perspective policy only.

${ }^{4}$ Only in the very special case, with $\log$-utility $\sigma=1$, and with $\eta=1$, and without shocks, $\hat{X}$ will disappear, see Gali and Monacelli (2002).
} 
The second group of terms from the welfare function involve the preference shock:

$$
\mathcal{W}_{S}=-U_{c} C \hat{C} \frac{g}{\sigma} \hat{\xi}-Y v_{y} \hat{Y} \frac{h}{\psi} \hat{\xi}
$$

Note that in the special case of log utility, we have $g=h=0$, so $W_{S}=0$. Once again we have terms in output and consumption. Using the relationship above implies

$$
\begin{aligned}
\mathcal{W}_{S} & =-U_{c} C(\Phi \hat{Y}+\hat{X}) \frac{g}{\sigma} \hat{\xi}-Y v_{y} \hat{Y} \frac{h}{\psi} \hat{\xi}+\operatorname{tip}(3) \\
& =-\hat{Y} \hat{\xi}\left(U_{c} C \Phi \frac{g}{\sigma}+Y v_{y} \frac{h}{\psi}\right)+\operatorname{tip}(3)
\end{aligned}
$$

In this case the terms in $\hat{X}$, which are all order two or above, are multiplied by the preference shock, so they can be absorbed into the tip(3) term.

We can then use (22) to replace $\hat{\xi}$ by terms in natural variables. Recall that the natural level of a variable is the level that would occur if there was no nominal inertia or IRS distortion. In our model, natural variables will depend on $\hat{\xi}$ and $\hat{Y}^{*}$ alone. The mark up (wages over output prices) is unaffected by changes in $\hat{\xi}$ and $\hat{Y}^{*}$ (with flexible prices, it depends on the degree of monopoly together with any output subsidy), so we can write to first order

$$
\hat{y}^{n} \frac{1}{\psi}+\hat{C}^{n} \frac{1}{\sigma}+\alpha \hat{S}^{n}+\hat{\xi}\left(\frac{h}{\psi}+\frac{g}{\sigma}\right)=\operatorname{tip}(2)
$$

We can use this expression to substitute out for the preference shock in $\mathcal{W}_{S}$. (As $\hat{\xi}$ is multiplied by $\hat{Y}$, we can ignore second order terms in the equation above.) Before doing this, let us write the third component of $\mathcal{W}$, which involves quadratic terms in output and consumption:

$$
\mathcal{W}_{Q}=U_{c}(\cdot) C\left[\frac{1}{2}\left(1-\frac{1}{\sigma}\right) \hat{C}^{2}\right]-Y v_{y}(\cdot)\left[\frac{1}{2}\left(1+\frac{1}{\psi}\right) \hat{Y}^{2}\right]
$$

In the special case of logarithmic utility, we have $\sigma=1, \psi=-1$, so $\mathcal{W}_{Q}=0$.

We can now use the results of agents optimisation to simplify these three components of welfare. We noted that, in a closed economy, we could assume a subsidy such that $U_{c}=v_{y}$, which set $\mathcal{W}_{L}=0$. The obvious analogy for an open economy is to set the subsidy such that $U_{c} C \Phi=Y v_{y}$. The three components of welfare then become

$$
\begin{aligned}
\mathcal{W}_{L} & =\frac{Y v_{y}}{\Phi} \hat{X}+\operatorname{tip}(3) \\
\mathcal{W}_{S} & =Y v_{y} \hat{Y}\left(\hat{y}^{n} \frac{1}{\varphi}+\hat{C}^{n} \frac{1}{\sigma}+\alpha \hat{S}^{n}\right)+t i p(3) \\
& =Y v_{y}\left(\hat{y}^{n} \hat{Y} \frac{1}{\varphi}+\hat{C}^{n} \frac{\hat{C}}{\Phi} \frac{1}{\sigma}+\alpha \hat{S}^{n} \hat{Y}\right)+t i p(3) \\
\mathcal{W}_{Q} & =\frac{Y v_{y}(\cdot)}{2}\left[\frac{1}{\Phi}\left(1-\frac{1}{\sigma}\right) \hat{C}^{2}-\left(1+\frac{1}{\psi}\right) \hat{Y}^{2}\right]
\end{aligned}
$$


noting that natural variables are also tip. Now note that

$$
\left(\hat{Y}-\hat{Y}^{n}\right)^{2}=\hat{Y}^{2}-2 \hat{Y} \hat{Y}^{n}+t i p
$$

and similarly for consumption, so we can write

$$
\begin{aligned}
\mathcal{W}_{Q} & =\frac{Y v_{y}(\cdot)}{2}\left[\frac{1}{\Phi}\left(1-\frac{1}{\sigma}\right)\left(\hat{C}-\hat{C}^{n}\right)^{2}+\left(\frac{1}{\Phi}-\frac{1}{\Phi \sigma}\right) \hat{C} \hat{C}^{n}\right. \\
& \left.-\left(1+\frac{1}{\psi}\right)\left(\hat{Y}-\hat{Y}^{n}\right)^{2}-\left(1+\frac{1}{\psi}\right) \hat{Y} \hat{Y}^{n}\right]+t i p
\end{aligned}
$$

Combining this with $\mathcal{W}_{S}$ gives

$$
\begin{aligned}
\mathcal{W}_{Q}+\mathcal{W}_{S} & =\frac{Y v_{y}(\cdot)}{2}\left[\frac{1}{\Phi}\left(1-\frac{1}{\sigma}\right)\left(\hat{C}-\hat{C}^{n}\right)^{2}-\frac{2}{\Phi} \hat{C} \hat{C}^{n}\right. \\
& \left.-\left(1+\frac{1}{\psi}\right)\left(\hat{Y}-\hat{Y}^{n}\right)^{2}+2 \hat{Y} \hat{Y}^{n}+2 \alpha \hat{S}^{n} \hat{Y}\right]+t i p(3) \\
& =\frac{Y v_{y}(\cdot)}{2}\left[\frac{1}{\Phi}\left(1-\frac{1}{\sigma}\right)\left(\hat{C}-\hat{C}^{n}\right)^{2}\right. \\
& \left.-\left(1+\frac{1}{\psi}\right)\left(\hat{Y}-\hat{Y}^{n}\right)^{2}+2 \alpha \hat{S}^{n} \hat{Y}+2 \hat{Y}\left(\hat{Y}^{n}-\hat{C}^{n}\right)\right]+t i p(3)
\end{aligned}
$$

(In the closed economy case, $\Phi=1$ and $\alpha=0$, so this simplifies to just a quadratic term in the output/consumption gap.) We can then add in the first component to give

$$
\begin{aligned}
\mathcal{W}_{Q}+\mathcal{W}_{S}+\mathcal{W}_{L} & =\frac{Y v_{y}(\cdot)}{2}\left[\frac{1}{\Phi}\left(1-\frac{1}{\sigma}\right)\left(\hat{C}-\hat{C}^{n}\right)^{2}-\left(1+\frac{1}{\psi}\right)\left(\hat{Y}-\hat{Y}^{n}\right)^{2}\right. \\
& \left.+2 \alpha \hat{S}^{n} \hat{Y}+2 \hat{Y}\left(\hat{Y}^{n}-\hat{C}^{n}\right)+\frac{2}{\Phi} \hat{X}\right]+t i p(3)
\end{aligned}
$$

where

$$
\begin{aligned}
\hat{X} & =\frac{1}{2} \Phi\left(\hat{Y}-\hat{Y}^{n}\right)^{2}-\Phi \alpha^{2} \eta(2-\alpha)\left(\hat{S}-\hat{S}^{n}\right)^{2}-\frac{1}{2} \frac{\Phi}{\Phi_{b}}\left(\hat{C}-\hat{C}^{n}\right)^{2} \\
& -\Phi \frac{\alpha \eta}{\sigma}((2-\alpha)+\sigma(1-\alpha))\left(\hat{S}-\hat{S}^{n}\right)\left(\hat{C}-\hat{C}^{n}\right) \\
& -\Phi \alpha \eta\left(\hat{Y}^{*}+2 \alpha(2-\alpha) \hat{S}^{n}+\frac{((2-\alpha)+\sigma(1-\alpha))}{\sigma} \hat{C}^{n}\right) \hat{S} \\
& +\Phi \hat{Y} \hat{Y}^{n}-\Phi\left(\frac{1}{\Phi_{b}} \hat{C}^{n}+\frac{\alpha \eta}{\sigma}((2-\alpha)+\sigma(1-\alpha)) \hat{S}^{n}\right) \hat{C}+\operatorname{tip}(3)
\end{aligned}
$$

We can now see that, in an open economy, welfare cannot be expressed in terms of gap variables alone. The minimum value for welfare will not in general be the point in which output and consumption gaps are zero. This is because the size of the monopolistic distortion in an open economy depends on changes in exogenous variables or preference shocks, which in turn influence the value of natural variables. While a constant subsidy can eliminate the monopolistic distortion for $\hat{Y}^{*}=\hat{\xi}=0$, it cannot do so for any other combination except by chance. To achieve this would require a subsidy that was conditional on these terms. (This point that has been noted by Beningno and Benigno (2003) in a related context.) 
We can now turn to the other question we needed to address, which was whether the welfare expression can only be written in terms of output alone. As we noted at the end of the previous section, the consumption and output gaps will only move together when the IRS distortion is absent i.e. $\hat{\zeta}=0$. Only in this special case (which is implicitly the case considered by Clarida, Gali, and Gertler (2001)) will it be possible to express welfare as a function of a single gap term. However, it is possible to substitute out the consumption gap in terms of the output gap and the terms of trade gap. The Appendix shows that we can obtain an expression for total welfare of the form ${ }^{5}$

$$
\begin{aligned}
\mathcal{W} & =C u_{C}\left\{A_{1}\left(\hat{Y}-\hat{Y}^{n}\right)^{2}+A_{2}\left(\hat{S}-\hat{S}^{n}\right)^{2}+A_{3}\left(\hat{S}-\hat{S}^{n}\right)\left(\hat{Y}-\hat{Y}^{n}\right)\right. \\
& +\hat{S}\left(A_{41} \hat{Y}^{n}+A_{42} \hat{S}^{n}+A_{45} \hat{Y}^{*}\right) \\
& \left.+\hat{Y}\left(A_{51} \hat{Y}^{n}+A_{52} \hat{S}^{n}+A_{55} \hat{Y}^{*}\right)+A_{6} \pi_{H}^{2}\right\}+\operatorname{tip}(3)
\end{aligned}
$$

Thus we can reduce the policy problem to one involving gaps in two variables, output and the terms of trade, plus inflation, but where quadratic gap terms are accompanied by linear gap terms multiplying natural values. ${ }^{6}$ The appendix also shows that we obtain a similar structure utilising the approach of Sutherland (2002b) and Benigno and Woodford (2004). A similar expression is stated in De Paoli (2004). Although the model has some similarities to ours, the IRS distortion is missing, there are no preference shocks and no derivation is provided.

The quadratic gap terms arise partly from $W_{Q}$, but also because of second order terms in the demand or international risk sharing equations entering through $W_{L}$. Thus, terms in the exchange rate gap arise even in the special case of log utility. Given the demand curve, it would of course also be possible to write an expression in the output and consumption gaps, without any term in the exchange rate gap. However, while policy has some experience in targeting the exchange rate, there are few examples where policy has explicitly targeted a consumption gap.

A key point to note about the terms of trade term is that, like output, it is in the form of deviations from the natural level i.e. the terms of trade that would occur with no nominal inertia or IRS shocks. A number of studies have experimented with simple feedback rules which include some form of exchange rate targeting, but generally not in terms of deviations from its natural level. For example, Kollmann (2002) finds that adding a quadratic term in the change in exchange rate to a feedback rule (with optimised parameters) that already includes output price inflation and output disequilibrium terms adds virtually nothing to welfare. ${ }^{7}$ This result

\footnotetext{
${ }^{5}$ It would, of course, be trivially possible to redefine gap variables such that it was no longer related to natural level, but was a function of other disturbances $\left(\hat{Y}^{n}, \hat{S}^{n}, \hat{Y}^{*}\right)$ as well. Formula (60) would contain the same quadratic terms, but would not contain linear terms, as in Benigno and Woodford (2004). The standard meaning of gaps would be lost in this case.

${ }^{6}$ Experiments with some alternative calibrations suggest that the magnitude of $A_{2}$ is less than $A_{1}$, but still significant, particularly bearing in mind that exchange rates are more volatile than output.

${ }^{7}$ In Kollmann (2003), it is argued that exchange rate targeting may be of greater value if it helps reduce the
} 
is interesting, because CPI inflation targeting can be roughly 'recovered' from separate terms on output price inflation and the change in the exchange rate. However our analysis suggests terms in exchange rate 'gap': the difference between actual exchange rate disequilibrium and the disequilibrium that would occur with no distortions. Not only is the dimension of this expression different from the change in the exchange rate, but a change in exchange rate term makes no attempt to allow for 'warranted' exchange rate movements i.e. natural disequilibrium. ${ }^{8}$

One early example that does come close to trying to capture the concept of an exchange rate gap is the Target Zone proposal of Williamson and Miller (Williamson and Miller (1987), see also Currie and Wren-Lewis (1989) for an evaluation), where interest rates differentials were assigned entirely to stabilising the real exchange rate around its medium term equilibrium level (FEER), and fiscal policy was assigned to inflation stabilisation. While this particular policy assignment may no longer be on the agenda (except, perhaps, for countries within a currency union), the FEER measure of an equilibrium exchange rate is close to the idea of a natural level. In particular, the FEER is the real exchange rate that would occur if the economy was in 'internal balance', which can be interpreted as abstracting from business cycle effects generated by nominal inertia as well as IRS shocks. Crucially the FEER is influenced by medium term net saving (private or public), which can be thought of as one example of a preference shock. In this sense the FEER is more like the natural level of the real exchange rate than PPP.

It is important, however, to recognise that our analysis does not imply that policy should always aim to eliminate exchange rate gaps. Some exchange rate gap may well be necessary to avoid large output gaps. Typically a tight monetary policy, responding to a positive output gap, will lead to an appreciation, which will a helpful element in the transmission mechanism. Where the exchange rate gap term plays an essential role is if a positive demand shock is combined with an excess appreciation as a result of some distortion to international risk sharing or uncovered interest parity. In this case the appreciation may eliminate the impact of the demand shock on the output gap, but a consumption gap would remain, and so a cut in interest rates to moderate the appreciation will enhance welfare.

\section{Conclusions}

In this paper we have examined the proposition in some recent papers that the objective function of a benevolent monetary policy maker in an open economy is identical to that for a closed economy. We have shown that this is no longer the case once we introduce a wider range of shocks into the model. Such shocks introduce a potential role for the terms of trade or exchange size of IRS shocks.

\footnotetext{
${ }^{8}$ For this reason CPI inflation targeting cannot be considered as an attempt to combine the output price inflation targeting and exchange rate targeting in this welfare framework.
} 
rate gap in the objective function alongside the output gap and output price inflation. This is an important result, because it shows for the first time why the central bank should be concerned with exchange rate disequilibria as well as output disequilibria because both impact directly on agents' utility. ${ }^{9}$ The intuition behind the result is straightforward. Unless there are no shocks to international risk sharing or uncovered interest parity, output gaps and consumption gaps may not move together. Welfare depends on both gaps. If central banks do not monitor the consumption gap directly, then they need to consider the exchange rate gap alongside the output gap in setting policy.

\footnotetext{
${ }^{9}$ Currie and Wren-Lewis (1989) place an exchange rate disequilibrium (from the FEER) term in an objective function, but their informal justification was rather different. They argued that this term could capture costs due to dispersion in traded/non-traded acticity. In the model in our paper all firms trade equally, so this issue does not arise, but it would be interesting to formally derive social welfare functions in models that did make this distinction.
} 


\section{References}

Aoki, K. (2002): "Optimal Monetary Policy Response to Relative Price Changes," Journal of Monetary Economics, 48, 55-80.

Batini, N., R. Harrison, and S. Millard (2003): "Monetary policy rules for an open economy," Journal of Economic Dynamics and Control, 27, 2059-2094.

Benigno, P., and M. Woodford (2004): "Inflation Stabilization and Welfare: The Case of a Distorted Steady State," mimeo, Princeton University.

Beningno, G., and P. Benigno (2003): "Price Stability in Open Economies," Review of Economic Studies, 70, 743-764.

Buiter, W., and C. Grafe (2003): "EMU or Ostrich," in Submissions on EMU from Leading Academics. H.M.Treasury.

Clarida, R., J. Gali, and M. Gertler (2001): "Optimal Monetary Policy in Open versus Closed Economies: An Integrated Approach," American Economic Review, 91, 248-252.

Currie, D., and S. Wren-Lewis (1989): "Evaluating blueprints for the conduct of international macropolicy," American Economic Review, 79, 264-269.

De Paoli, B. (2004): "Monetary Policy and Welfare in a Small Open Economy," Discussion paper, LSE, CEP discussion paper 639.

Gali, J., and T. Monacelli (2002): "Monetary Policy and Exchange Rate Volatility in a Small Open Economy," Working Paper 8905, NBER.

Kollmann, R. (2002): "Monetary Policy in An Open Economy: Effects on Welfare and Business Cycles," Journal of Monetary Economics, 49, 989-1015.

— (2003): "Monetary Policy Rules in an Interdependent World," Mimeo.

PAPPA, E. (2002): "Do the ECB and the Fed really need to Cooperate? Optimal Monetary Policy in a Two-Country World," Mimeo, LSE.

Rotemberg, J. J., And M. Woodford (1997): "An Optimization-based Econometric Framework for the Evaluation of Monetary Policy," in NBER Macroeconomics Annual, pp. 297-344.

Steinsson, J. (2002): "Optimal Monetary Policy in an Economy with Inflation Presistence," mimeo, Harvard University, available at http://www.people.fas.harvard.edu/ steinss/papers/STjme.pdf. 
Sutherland, A. (2002a): "Cost-Push Shocks and Monetary Policy in Open Economies," mimeo, University of St.Andrews.

Sutherland, A. (2002b): "A Simple Second-Order Solution Method For Dynamic General Equilibrium Models," Discussion paper, CEPR discussion paper 3554.

Williamson, J., and M. Miller (1987): Target Zones and Indicators: A Blueprint for the International Coordination of Economic Policy. Institute for International Economics.

Woodford, M. (2003): Interest and Prices: Foundations of a Theory of Monetary Policy. Princeton University Press, Princeton, NJ. 


\section{A Second order approximation to key economic relationships}

\section{A.1 Risk Sharing Condition}

From the first order conditions, (17) and the similar for the other country:

$$
\beta \frac{u_{C}\left(C_{t+1}^{*}, \xi_{t+1}\right)}{u_{C}\left(C_{t}^{*}, \xi_{t}\right)} \frac{P_{t}^{*} E_{t} \phi\left(\zeta_{t}\right)}{P_{t+1}^{*} E_{t+1} \phi\left(\zeta_{t+1}\right)}=\frac{1}{\left(1+i_{t}\right)}
$$

it follows

$$
\frac{u_{C}\left(C_{t+1}, \xi_{t+1}\right) u_{C}\left(C_{t}^{*}, \xi_{t}\right)}{u_{C}\left(C_{t+1}^{*}, \xi_{t+1}\right) u_{C}\left(C_{t}, \xi_{t}\right)} \frac{\phi\left(\zeta_{t}\right)}{\phi\left(\zeta_{t+1}\right)}=\frac{Q_{t}}{Q_{t+1}}
$$

where the real exchange rate $Q_{t}$ is defined in (11). Assuming some initial conditions $\vartheta$ we can iterate (62) and obtain (Gali and Monacelli (2002)):

$$
u_{C}\left(C_{t}^{*}, \xi_{t}\right) \phi\left(\zeta_{t}\right)=\vartheta Q_{t} u_{C}\left(C_{t}, \xi_{t}\right)
$$

The second order linearisation yields:

$$
\begin{aligned}
u_{C}\left(C_{s}^{i}, \xi_{s}\right) & =u_{C}(C, 1)+u_{C C}(C, 1) C\left(\left(\hat{C}_{s}+\frac{1}{2} b \hat{C}_{s}^{2}\right)+g\left(\hat{\xi}_{s}+\frac{1}{2} a \hat{\xi}_{s}^{2}\right)\right. \\
& \left.+d \hat{C}_{s} \hat{\xi}_{s}\right)+o\left(C_{s}, \xi_{s}\right)^{2} \\
u_{C}\left(C_{s}^{* i}, \xi_{s}\right) & =u_{C}\left(C^{*}, 1\right)+u_{C C}\left(C^{*}, 1\right) C^{*}\left(\left(\hat{C}_{s}^{*}+\frac{1}{2} b \hat{C}_{s}^{* 2}\right)+g\left(\hat{\xi}_{s}^{*}+\frac{1}{2} a \hat{\xi}_{s}^{* 2}\right)\right. \\
& \left.+d \hat{C}_{s}^{*} \hat{\xi}_{s}^{*}\right)+o\left(C_{s}^{*}, \xi_{s}^{*}\right)^{2}
\end{aligned}
$$

Therefore, we substitute these relationships into (63) and, using $u_{C}\left(C^{*}, 1\right)=\vartheta Q u_{C}(C, 1)$, obtain the following relationship, where all terms of order higher than two are eliminated:

$$
\begin{aligned}
& \hat{C}_{s}=\hat{C}_{s}^{*}+\sigma \hat{Q}_{t}-\sigma \hat{\zeta}_{s}-g\left(\hat{\xi}_{s}-\hat{\xi}_{s}^{*}\right) \\
& -\frac{1}{2} b \hat{C}_{s}^{2}-d \hat{C}_{s} \hat{\xi}_{s}+\frac{1}{2} \sigma \hat{Q}_{t}^{2}+\frac{1}{2} b \hat{C}_{s}^{* 2}-\hat{C}_{s} \hat{Q}_{t}-g \hat{\xi}_{s} \hat{Q}_{t} \\
& -\frac{1}{2} g a \hat{\xi}_{s}^{2}-\frac{1}{2} \sigma \hat{\zeta}_{s}^{2}+\hat{C}_{s}^{*} \hat{\zeta}_{s}+g \hat{\xi}_{s}^{*} \hat{\zeta}_{s}+\frac{1}{2} g a \hat{\xi}_{s}^{* 2}+d \hat{C}_{s}^{*} \hat{\xi}_{s}^{*}
\end{aligned}
$$

the linear version of which is

$$
\hat{C}_{s}=\hat{C}_{s}^{*}+\sigma \hat{Q}_{t}-\sigma \hat{\zeta}_{s}-g\left(\hat{\xi}_{s}-\hat{\xi}_{s}^{*}\right)
$$

It is convenient to write down the formula in terms of trade, rather than real exchange rate. The real exchange rate is defined in (11):

$$
Q=\frac{E P^{*}}{P}=\frac{E P^{*}}{P_{H}} \frac{P_{H}}{P}=\frac{P_{F}}{P_{H}} \frac{P_{H}}{P}=S\left((1-\alpha)+\alpha S^{(1-\eta)}\right)^{-\frac{1}{1-\eta}}
$$

Linearisation up to second order terms yields:

$$
\hat{Q}=\ln Q=\ln \left(S\left((1-\alpha)+\alpha S^{(1-\eta)}\right)^{-\frac{1}{1-\eta}}\right)=(1-\alpha) \hat{S}+\frac{1}{2}(1-\alpha)(\alpha(\eta-1)-1) \hat{S}^{2}
$$


So we can rewrite (64) as:

$$
\begin{aligned}
& \hat{C}_{s}=\hat{C}_{s}^{*}+\sigma(1-\alpha) \hat{S}+\frac{1}{2} \sigma \alpha(1-\alpha)(\eta-2) \hat{S}^{2}-\sigma \hat{\zeta}_{s}-g\left(\hat{\xi}_{s}-\hat{\xi}_{s}^{*}\right) \\
& -\frac{1}{2} b \hat{C}_{s}^{2}-d \hat{C}_{s} \hat{\xi}_{s}+\frac{1}{2} b \hat{C}_{s}^{* 2}-(1-\alpha) \hat{C_{s}} \hat{S}-(1-\alpha) g \hat{\xi}_{s} \hat{S} \\
& -\frac{1}{2} g a \hat{\xi}_{s}^{2}-\frac{1}{2} \sigma \hat{\zeta}_{s}^{2}+\hat{C}_{s}^{*} \hat{\zeta}_{s}+g \hat{\xi}_{s}^{*} \hat{\zeta}_{s}+\frac{1}{2} g a \hat{\xi}_{s}^{* 2}+d \hat{C}_{s}^{*} \hat{\xi}_{s}^{*}
\end{aligned}
$$

This is a general formula, which assumes no restrictions on the form of utility function and the model parameters.

\section{A.2 Aggregate Demand}

Aggregation implies:

$$
Y=C_{H}+C_{H}^{*}
$$

if we linearise it we get

$$
\begin{aligned}
\hat{Y}+\frac{1}{2} \hat{Y}^{2} & =(1-\alpha)\left(\hat{C}_{H}+\frac{1}{2} \hat{C}_{H}^{2}\right)+\alpha\left(\hat{C}_{H}^{*}+\frac{1}{2} \hat{C}_{H}^{* 2}\right) \\
\hat{Y} & =(1-\alpha)\left(\hat{C}_{H}+\frac{1}{2} \hat{C}_{H}^{2}\right)+\alpha\left(\hat{C}_{H}^{*}+\frac{1}{2} \hat{C}_{H}^{* 2}\right)-\frac{1}{2} \hat{Y}^{2}
\end{aligned}
$$

On the other hand,

$$
C=C_{H}+C_{F}
$$

and linearisation gives:

$$
\hat{C}=(1-\alpha)\left(\hat{C}+\frac{1}{2} \hat{C}_{H}^{2}\right)+\alpha\left(\hat{C}_{F}+\frac{1}{2} \hat{C}_{F}^{2}\right)-\frac{1}{2} \hat{C}^{2}
$$

Now,

$$
\begin{aligned}
\hat{Y}_{s} & =\hat{C}+\alpha\left(\left(\hat{C}_{H}^{*}-\hat{C}^{*}\right)-\left(\hat{C}_{F}-\hat{C}\right)\right) \\
& +\alpha \frac{1}{2}\left(\left(\hat{C}_{H}^{* 2}-\hat{C}^{* 2}\right)-\left(\hat{C}_{F}^{2}-\hat{C}^{2}\right)\right) \\
& +\frac{1}{2} \hat{C}^{2}-\frac{1}{2} \hat{Y}^{2}+\alpha \hat{C}^{*}-\alpha \hat{C}+\alpha \frac{1}{2} \hat{C}^{* 2}-\alpha \frac{1}{2} \hat{C}^{2}
\end{aligned}
$$

From (6) it follows

$$
\begin{aligned}
& \hat{C}_{H}^{*}-\hat{C}^{*}=-\eta\left(P_{H}^{*}-P^{*}\right), \quad \hat{C}_{F}-\hat{C}=-\eta\left(P_{F}-P\right) \\
& \hat{P}_{H}-\hat{P}=-\alpha \hat{S}, \hat{P}_{F}-\hat{P}=(1-\alpha) \hat{S}, \hat{P}_{H}^{*}-\hat{P}^{*}=-\alpha^{*} \hat{S} \approx-\hat{S}
\end{aligned}
$$


and

$$
\begin{aligned}
\hat{Y}_{s} & =(1-\alpha) \hat{C}+\alpha \hat{C}^{*}+\alpha \eta(2-\alpha) \hat{S}+\frac{1}{2} \eta^{2} \alpha^{2}(2-\alpha) \hat{S}^{2} \\
& +\alpha \eta \hat{S} \hat{C}^{*}+\alpha \eta(1-\alpha) \hat{S} \hat{C}+\frac{1}{2}(1-\alpha) \hat{C}^{2}-\frac{1}{2} \hat{Y}^{2}+\frac{1}{2} \alpha \hat{C}^{* 2}
\end{aligned}
$$

where the linear version can be written as:

$$
\hat{Y}_{s}=(1-\alpha) \hat{C}+\alpha \hat{C}^{*}+\alpha \eta(2-\alpha) \hat{S}
$$

Again, formula (70) assumes no restrictions on the form of utility function and the model parameters.

\section{A.3 Phillips Curve}

As it is shown in Woodford (2003), the problem of profit maximisation for the private sector leads to the following first order condition:

$$
0=\mathcal{E}_{t} \sum_{s=t}^{\infty} \gamma^{s-t} R_{t, s} Y_{s}\left(\frac{p_{H, t}(z)}{P_{H, s}}\right)^{-\epsilon}\left[p_{H, t}(z)-\mu P_{s} \frac{v_{y}\left(\left(\frac{p_{H, t}(z)}{P_{H, s}}\right)^{-\epsilon} Y_{s}, \xi_{s}\right)}{u_{C}\left(C_{s}^{i}, \xi_{s}\right)}\right]
$$

which is an equation for price determination.

Here, we assume an iso-elastic form of both components of the household utility function. Namely we assume that

$$
v(y(z), \xi)=\frac{y(z)^{1+\frac{1}{\psi}} \xi^{-\frac{1}{\psi}}}{1+\frac{1}{\psi}}, u(C, \xi)=\frac{C^{1-\frac{1}{\sigma}} \xi^{\frac{1}{\sigma}}}{1-\frac{1}{\sigma}}
$$

therefore

$$
\sigma=-\frac{u_{C}(y(z), \xi)}{u_{C C}(y(z), \xi) C}, \psi=\frac{v_{y}(y(z), \xi)}{v_{y y}(y(z), \xi) y(z)}
$$

and, for the future reference

$$
\begin{aligned}
& a=1+\frac{u_{C \xi \xi}(C, 1)}{u_{C \xi}(C, 1)}=\frac{1}{\sigma}, b=1+\frac{C u_{C C C}(C, 1)}{u_{C C}(C, 1)}=-\frac{1}{\sigma} \\
& d=\frac{u_{C C \xi}(C, 1)}{u_{C C}(C, 1)}=\frac{1}{\sigma}, g=\frac{u_{C \xi}(C, 1)}{C u_{C C}(C, 1)}=-1, h=\frac{v_{y \xi}(Y, 1)}{v_{y y}(Y, 1) Y}=-1
\end{aligned}
$$

Note that for iso-elastic utility

$$
\sigma d+g=0
$$


This assumption allows us to derive optimal price as an explicit function of all other parameters. Substitute utility in (72) and collecting similar terms we get:

$$
\begin{aligned}
& \mathcal{E}_{t} \sum_{s=t}^{\infty} \gamma^{s-t} R_{t, s} Y_{s} P_{H, s}\left(\frac{P_{H, t}}{P_{H, s}}\right)^{-\epsilon+1} \\
& =\left(\frac{p_{H, t}(z)}{P_{H, t}}\right)^{-\frac{\epsilon}{\psi}-1} \mathcal{E}_{t} \sum_{s=t}^{\infty} \gamma^{s-t} R_{t, s} \mu Y_{s} P_{H, s} \frac{P_{s}}{P_{H, s}} \frac{Y_{s}^{\frac{1}{\psi}} \xi_{s}^{-\frac{1}{\psi}}}{C_{s}^{-\frac{1}{\sigma}} \xi_{s}^{\frac{1}{\sigma}}}\left(\frac{P_{H, t}}{P_{H, s}}\right)^{-\frac{\epsilon}{\psi}-\epsilon}
\end{aligned}
$$

from where:

$$
\frac{p_{H, t}(z)}{P_{H, t}}=\left(\frac{\mathcal{E}_{t} \sum_{s=t}^{\infty} \gamma^{s-t} R_{t, s} Y_{s} P_{H, s}\left(\frac{P_{H, t}}{P_{H, s}}\right)^{-\epsilon+1}}{\mathcal{E}_{t} \sum_{s=t}^{\infty} \gamma^{s-t} R_{t, s} \mu Y_{s} P_{H, s} \frac{P_{s}}{P_{H, s}} \frac{Y_{s}^{\frac{1}{\psi}} \xi_{s}^{-\frac{1}{\psi}}}{C_{s}^{-\frac{1}{\sigma}} \xi_{s}^{\frac{1}{\sigma}}}\left(\frac{P_{H, t}}{P_{H, s}}\right)^{-\frac{\epsilon}{\psi}-\epsilon}}\right)^{\frac{1}{-\frac{\epsilon}{\psi}-1}}
$$

Recalling that first order conditions for consumption optimisation leads to

$$
\beta^{s-t} \frac{u_{C}\left(C_{s}, \xi_{s}\right)}{u_{C}\left(C_{t}, \xi_{t}\right)} \frac{P_{t}}{P_{s}}=R_{t, s}
$$

and, substituting out $R_{t, s}$ we obtain

$$
\frac{p_{H, t}(z)}{P_{H, t}}=\left(\frac{\mu \mathcal{E}_{t} \sum_{s=t}^{\infty}(\gamma \beta)^{s-t} Y_{s} v_{y}\left(Y_{s}, \xi_{s}\right)\left(\frac{P_{H, t}}{P_{H, s}}\right)^{-\left(\frac{1}{\psi}+1\right) \epsilon}}{\mathcal{E}_{t} \sum_{s=t}^{\infty}(\gamma \beta)^{s-t} Y_{s} u_{C}\left(C_{s}, \xi_{s}\right) \frac{P_{H, s}}{P_{s}}\left(\frac{P_{H, t}}{P_{H, s}}\right)^{1-\epsilon}}\right)^{\frac{1}{\psi}+1}=\left(\frac{K_{t}}{F_{t}}\right)^{\omega}
$$

where

$$
\begin{aligned}
K_{t} & =\mathcal{E}_{t} \sum_{s=t}^{\infty}(\gamma \beta)^{s-t} Y_{s} v \mu_{y}\left(Y_{s}, \xi_{s}\right)\left(\frac{P_{H, t}}{P_{H, s}}\right)^{-\left(\frac{1}{\psi}+1\right) \epsilon}=\mathcal{E}_{t} \sum_{s=t}^{\infty}(\gamma \beta)^{s-t} k_{s, t} \\
F_{t} & =\mathcal{E}_{t} \sum_{s=t}^{\infty}(\gamma \beta)^{s-t} Y_{s} u_{C}\left(C_{s}, \xi_{s}\right) \frac{P_{H, s}}{P_{s}}\left(\frac{P_{H, t}}{P_{H, s}}\right)^{1-\epsilon}=\mathcal{E}_{t} \sum_{s=t}^{\infty}(\gamma \beta)^{s-t} f_{s, t}
\end{aligned}
$$

and

$$
\begin{aligned}
& f_{s, t}=u_{C}\left(C_{s}, \xi_{s}\right) \frac{P_{H, s}}{P_{s}} Y_{s}\left(\frac{P_{H, t}}{P_{H, s}}\right)^{1-\epsilon} \\
& k_{s, t}=\mu v_{y}\left(Y_{s}, \xi_{s}\right) Y_{s}\left(\frac{P_{H, t}}{P_{H, s}}\right)^{-\left(\frac{1}{\psi}+1\right) \epsilon} \\
& \omega=\frac{\psi}{\epsilon+\psi} .
\end{aligned}
$$

The only difference from Benigno and Woodford (2004) is that we distinguish $P_{H, t}$ and $P_{t}$.

With second order approximation of

$$
\begin{aligned}
\hat{F}_{t}+\frac{1}{2} \hat{F}_{t}^{2} & =(1-\gamma \beta) \mathcal{E}_{t} \sum_{s=t}^{\infty}(\gamma \beta)^{s-t}\left(\hat{f}_{s, t}+\frac{1}{2} \hat{f}_{s, t}^{2}\right) \\
\hat{K}_{t}+\frac{1}{2} \hat{K}_{t}^{2} & =(1-\gamma \beta) \mathcal{E}_{t} \sum_{s=t}^{\infty}(\gamma \beta)^{s-t}\left(\hat{k}_{s, t}+\frac{1}{2} \hat{k}_{s, t}^{2}\right)
\end{aligned}
$$


a log-linearised (up to second order) version of (76) can be written as

$$
\begin{aligned}
\frac{1}{\omega} \ln \frac{p_{H, t}(z)}{P_{H, t}} & =(1-\gamma \beta) \mathcal{E}_{t} \sum_{s=t}^{\infty}(\gamma \beta)^{s-t}\left(\hat{k}_{s, t}-\hat{f}_{s, t}\right) \\
& +\frac{(1-\gamma \beta)}{2} \mathcal{E}_{t} \sum_{s=t}^{\infty}(\gamma \beta)^{s-t}\left(\hat{k}_{s, t}^{2}-\hat{f}_{s, t}^{2}\right)+\frac{1}{2}\left(\hat{F}_{t}-\hat{K}_{t}\right)\left(\hat{F}_{t}+\hat{K}_{t}\right)
\end{aligned}
$$

where, by definition of $k_{s, t}$ and $f_{s, t}$ :

$$
\begin{aligned}
\hat{k}_{s, t}-\hat{f}_{s, t} & =\frac{1}{\psi} \ln \left(\frac{Y_{s}}{Y}\right)+\frac{1}{\sigma} \ln \left(\frac{C_{s}}{C}\right)-\left(\frac{1}{\sigma}+\frac{1}{\psi}\right) \ln \xi_{s} \\
- & {\left[\frac{\epsilon}{\psi}+1\right] \ln \left(\frac{P_{H, t}}{P_{H, s}}\right)-\ln \frac{P_{H, s}}{P_{s}} } \\
\hat{k}_{s, t}+\hat{f}_{s, t} & =\left[1-\left(\frac{1}{\psi}+2\right) \epsilon\right] \ln \left(\frac{P_{H, t}}{P_{H, s}}\right)+X_{s} \\
X_{s} & =\left(\frac{1}{\psi}+2\right) \ln \left(\frac{Y_{s}}{Y}\right)-\frac{1}{\sigma} \ln \left(\frac{C_{s}}{C}\right)+\left(\frac{1}{\sigma}-\frac{1}{\psi}\right) \ln \xi_{s}+\ln \frac{P_{H, s}}{P_{s}}
\end{aligned}
$$

Substituting these expressions back to (77), we obtain

$$
\begin{aligned}
\ln \frac{p_{H, t}(z)}{P_{H, t}} & =\omega(1-\gamma \beta) \mathcal{E}_{t} \sum_{s=t}^{\infty}(\gamma \beta)^{s-t}\left(\hat{k}_{s, t}-\hat{f}_{s, t}\right) \\
& +\frac{\omega(1-\gamma \beta)}{2} \mathcal{E}_{t} \sum_{s=t}^{\infty}(\gamma \beta)^{s-t}\left(\frac{1}{\psi} \ln \left(\frac{Y_{s}}{Y}\right)+\frac{1}{\sigma} \ln \left(\frac{C_{s}}{C}\right)\right. \\
& \left.-\left(\frac{1}{\sigma}+\frac{1}{\psi}\right) \ln \xi_{s}-\ln \frac{P_{H, s}}{P_{s}}\right)\left(X_{s}+\left[1-\left(\frac{1}{\psi}+2\right) \epsilon\right] \ln \left(\frac{P_{H, t}}{P_{H, s}}\right)\right) \\
& -\frac{\omega(1-\gamma \beta)}{2}\left[\frac{\epsilon}{\psi}+1\right] \mathcal{E}_{t} \sum_{s=t}^{\infty}(\gamma \beta)^{s-t}\left(X_{s}+\left[1-\left(\frac{1}{\psi}+2\right) \epsilon\right] \ln \left(\frac{P_{H, t}}{P_{H, s}}\right)\right) \ln \left(\frac{P_{H, t}}{P_{H, s}}\right) \\
& -\frac{(1-\gamma \beta)}{2} \ln \frac{p_{H, t}(z)}{P_{H, t}} \mathcal{E}_{t} \sum_{s=t}^{\infty}(\gamma \beta)^{s-t}\left(X_{s}+\left[1-\left(\frac{1}{\psi}+2\right) \epsilon\right] \ln \left(\frac{P_{H, t}}{P_{H, s}}\right)\right)
\end{aligned}
$$

Using the notation:

$$
\begin{aligned}
Z_{t} & =\sum_{s=t}^{\infty}(\gamma \beta)^{s-t}\left(X_{s}+\left[1-\left(\frac{1}{\psi}+2\right) \epsilon\right] \ln \left(\frac{P_{H, t}}{P_{H, s}}\right)\right) \\
z_{s} & =\hat{k}_{s, t}-\hat{f}_{s, t}+\frac{1}{\omega} \ln \left(\frac{P_{H, t}}{P_{H, s}}\right)
\end{aligned}
$$

we can write (as Benigno and Woodford (2004) do)

$$
\begin{aligned}
& \frac{1}{\omega(1-\gamma \beta)} \ln \frac{p_{H, t}(z)}{P_{H, t}} \\
& =z_{t}+\frac{\gamma \beta}{\omega(1-\gamma \beta)}\left[\ln \frac{p_{H, t+1}(z)}{P_{H, t+1}}-\ln \frac{P_{H, t}}{P_{H, t+1}}\right]+\frac{1}{2} z_{t} X_{t} \\
& -\frac{1}{2 \omega} \ln \frac{p_{H, t}(z)}{P_{H, t}} Z_{t}+\frac{\gamma \beta}{2 \omega} \mathcal{E}_{t}\left[\ln \frac{p_{H, t+1}(z)}{P_{H, t+1}}-\ln \left(\frac{P_{H, t}}{P_{H, t+1}}\right)\right] Z_{t+1} \\
& +\frac{\gamma \beta\left[1-\left(\frac{1}{\psi}+2\right) \epsilon\right]}{2} \frac{1}{\omega(1-\gamma \beta)} \mathcal{E}_{t} \ln \frac{P_{H, t}}{P_{H, t+1}}\left[\ln \frac{p_{H, t+1}(z)}{P_{H, t+1}}-\ln \frac{P_{H, t}}{P_{H, t+1}}\right]
\end{aligned}
$$


and, using that

$$
\ln \frac{p_{H, t}(z)}{P_{H, t}}=\frac{\gamma}{(1-\gamma)} \pi_{H, t}-\frac{1}{2} \frac{\gamma(1-\epsilon)}{(1-\gamma)^{2}} \pi_{H, t}^{2}
$$

we finally obtain

$$
\begin{aligned}
\pi_{H, t} & =\beta \pi_{H, t+1}+\frac{\omega(1-\gamma \beta)(1-\gamma)}{\gamma} z_{t} \\
& +\frac{1}{2} \frac{(1-\epsilon)}{(1-\gamma)} \pi_{H, t}^{2}+\frac{1}{2} \frac{\omega(1-\gamma \beta)(1-\gamma)}{\gamma} z_{t} X_{t} \\
& -\frac{(1-\gamma \beta)}{2} \pi_{H, t} Z_{t}+\frac{\beta(1-\gamma \beta)}{2} \pi_{H, t+1} Z_{t+1}-\frac{\beta}{2} \frac{\gamma \epsilon\left(\frac{1}{\psi}+1\right)}{(1-\gamma)} \pi_{H, t+1}^{2}
\end{aligned}
$$

It is clear that the first-order approximation to the price-setting equation can be written as a conventional Phillips curve:

$$
\pi_{H, t}=\beta \pi_{H, t+1}+\kappa z_{t}
$$

where

$$
\kappa=\frac{\omega(1-\gamma \beta)(1-\gamma)}{\gamma}=\frac{\psi(1-\gamma \beta)(1-\gamma)}{(\epsilon+\psi) \gamma}
$$

Denote

$$
V_{t}=\pi_{H, t}-\frac{1}{2} \frac{\gamma \epsilon\left[\frac{1}{\psi}+1\right]}{(1-\gamma)} \pi_{H, t}^{2}+\frac{(1-\gamma \beta)}{2} \pi_{H, t} Z_{t}
$$

then we can rewrite $(78)$ as

$$
V_{t}=\beta V_{t+1}+\kappa z_{t}+\frac{1}{2} \kappa z_{t} X_{t}+\frac{1}{2} \frac{(1-\epsilon)-\gamma \epsilon\left(\frac{1}{\psi}+1\right)}{(1-\gamma)} \pi_{H, t}^{2}
$$

This formula is convenient for our future derivations, but we still need to define $z_{t}$ and $X_{t}$ in terms of economic variables. Recall that

$$
\ln \frac{P_{H, t}}{P_{t}}=-\alpha \hat{S}+\frac{1}{2} \alpha(\eta-1)(1-\alpha) \hat{S}^{2}
$$

is a second-order approximation to the relative price, so the final formulae for all terms in (80) are

$$
\begin{aligned}
z_{t} & =\frac{1}{\psi} \hat{Y}_{t}+\frac{1}{\sigma} \hat{C}_{t}-\left(\frac{1}{\sigma}+\frac{1}{\psi}\right) \hat{\xi}_{t}+\alpha \hat{S}_{t}-\frac{1}{2} \alpha(\eta-1)(1-\alpha) \hat{S}_{t}^{2} \\
X_{t} & =\left(\frac{1}{\psi}+2\right) \hat{Y}_{t}-\frac{1}{\sigma} \hat{C}_{t}+\left(\frac{1}{\sigma}-\frac{1}{\psi}\right) \hat{\xi}_{t}-\alpha \hat{S}_{t}+\frac{1}{2} \alpha(\eta-1)(1-\alpha) \hat{S}_{t}^{2}
\end{aligned}
$$

Integrating (80) forward

$$
V_{t_{0}}=\sum_{t=t_{0}}^{\infty} \beta^{t-t_{0}} \kappa\left(z_{t}+\frac{1}{2} z_{t} X_{t}\right)+\frac{\psi(1-\epsilon)-\gamma \epsilon(1+\psi)}{2 \psi(1-\gamma)} \sum_{t=t_{0}}^{\infty} \beta^{t-t_{0}} \pi_{H, t}^{2}
$$




\section{B Second Order Approximation to Utility}

Expanding $U\left(C_{s}, \xi_{s}\right)$ around $U(C, 1)$ gives

$$
\begin{aligned}
U\left(C_{s}, \xi_{s}\right) & =U_{C}(\cdot) C\left(\hat{C}_{s}+\frac{1}{2} \hat{C}_{s}^{2}\right)-\frac{1}{2} \frac{U_{C}(\cdot)}{\sigma} C \hat{C}_{s}^{2}-U_{C}(\cdot) \frac{g}{\sigma} C \hat{C}_{s} \hat{\xi}_{s}+\operatorname{tip}(3) \\
& =U_{c}(\cdot) C\left[\hat{C}_{s}+\frac{1}{2}\left(1-\frac{1}{\sigma}\right) \hat{C}_{s}^{2}-\frac{g}{\sigma} \hat{C}_{s} \hat{\xi}_{s}\right]+\operatorname{tip}(3)
\end{aligned}
$$

Applying the same procedure to $v\left(y_{s}(z), \xi_{s}\right)$ gives

$$
v\left(y_{s}(z), \xi_{s}\right)=v_{y}(\cdot) y\left[\hat{y}_{s}(z)+\frac{1}{2}\left(1+\frac{1}{\psi}\right) \hat{y}_{s}(z)^{2}+\frac{h}{\psi} \hat{y}_{s}(z) \hat{\xi}_{s}\right]+\operatorname{tip}(3)
$$

Integrating over goods

$$
\begin{aligned}
\int_{0}^{1} v\left(y_{s}(z), \xi_{s}\right) d z & =Y v_{y}(\cdot) \int_{0}^{1}\left[\hat{y}_{s}(z)+\frac{1}{2}\left(1+\frac{1}{\psi}\right) \hat{y}(z)^{2}+\frac{h}{\psi} \hat{y}(z) \hat{\xi}\right] d z+t i p(3) \\
& =Y v_{y}(\cdot)\left[E_{z} \hat{y}(z)+\frac{1}{2}\left(1+\frac{1}{\psi}\right)\left[\left(E_{z} \hat{y}(z)\right)^{2}\right.\right. \\
& \left.\left.+V_{z} \hat{y}(z)\right]+\frac{h}{\psi} \hat{\xi}_{s} E_{z} \hat{y}(z)\right]+\operatorname{tip}(3)
\end{aligned}
$$

where the expectation $E_{z} x=\int x d z$ and the variance $V_{z}=\int\left(x-E_{z} x\right)^{2}=\int x^{2} d z-E_{x} x$.

We can define an index of aggregate demand $Y$ as

$$
Y=\left[\int_{0}^{1} y(z)^{\frac{\epsilon-1}{\epsilon}} d z\right]^{\frac{\epsilon}{\epsilon-1}}
$$

where $\epsilon$ is the elasticity of demand. Taking a Taylor expansion of this yields

$$
\hat{Y}=E_{z} \hat{y}(z)+\frac{1}{2}\left(1-\frac{1}{\epsilon}\right) V_{z} \hat{y}(z)+O(3)
$$

We can use this to eliminate terms in $E_{z} \hat{y}(z)$, noting that $\left(E_{z} \hat{y}(z)\right)^{2}=\hat{Y}^{2}+O(|\xi|)^{3}$, and that $V_{z} \hat{y}(z) \hat{\xi}$ is $O(3)$. This gives

$$
\begin{aligned}
\int_{0}^{1} v\left(y_{s}(z), \xi_{s}\right) d z & =Y v_{y}(\cdot)\left[\hat{Y}_{s}-\frac{1}{2}\left(1-\frac{1}{\epsilon}\right) V_{z} \hat{y}(z)\right. \\
& \left.+\frac{1}{2}\left(1+\frac{1}{\psi}\right)\left[\hat{Y}_{s}^{2}+V_{z} \hat{y}(z)\right]+\frac{h}{\psi} \hat{\xi}_{s} \hat{Y}_{s}\right]+t i p(3) \\
& =Y v_{y}(\cdot)\left[\hat{Y}_{s}\left(1+\frac{h}{\psi} \hat{\xi}_{s}\right)+\frac{1}{2}\left(1+\frac{1}{\psi}\right) \hat{Y}_{s}^{2}\right. \\
& \left.+V_{z} \hat{y}(z) \frac{1}{2}\left(\frac{1}{\psi}+\frac{1}{\epsilon}\right)\right]+\operatorname{tip}(3)
\end{aligned}
$$


We can now combine the two calculations to give

$$
\begin{aligned}
\mathcal{W} & =U\left(C_{s}, \xi_{s}\right)-\int_{0}^{1} v\left(y_{s}(z), \xi_{s}\right) d z \\
& =U_{c}(\cdot) C\left[\hat{C}_{s}\left(1-\frac{g}{\sigma} \hat{\xi}_{s}\right)+\frac{1}{2}\left(1-\frac{1}{\sigma}\right) \hat{C}_{s}^{2}\right] \\
& -Y v_{y}(\cdot)\left[\hat{Y}_{s}\left(1+\frac{h}{\psi} \hat{\xi}_{s}\right)+\frac{1}{2}\left(1+\frac{1}{\psi}\right) \hat{Y}_{s}^{2}\right. \\
& \left.+V_{z} \hat{y}(z) \frac{1}{2}\left(\frac{1}{\psi}+\frac{1}{\epsilon}\right)\right]+t i p(3)
\end{aligned}
$$

This is equation (44) in the main text, but with time subscripts included.

Until now, these manipulations have only used one model property, which was the definition of the output index from the demand curve. As such, the analysis so far also applies to a closed economy, and is identical to that in Woodford (2003) and Steinsson (2002). We have four types of term in this expression: those involving level deviations in $C$ and $Y$, those combining these deviations with shocks, quadratic terms in deviations in $C$ and $Y$, and a term in the variance of output across goods. This last term can be related to the variance of individual prices using the demand curve: i.e. by taking logs of

$$
y(z)=\left(\frac{p_{H}(z)}{P_{H}}\right)^{-\epsilon} Y
$$

(which comes from adding consumption across home and overseas) it follows that with Calvo contracts this term can be represented as inflation squared (Rotemberg and Woodford (1997)):

$$
V_{z} \hat{y}(z)=\epsilon^{2} V_{x} \hat{p}(z)=\frac{\epsilon^{2} \gamma}{(1-\gamma \beta)(1-\gamma)} \pi_{H, t}^{2}
$$

Therefore, (88) becomes

$$
\begin{aligned}
W_{s} & =U_{c}(\cdot) C\left[\hat{C}_{s}\left(1-\frac{g}{\sigma} \hat{\xi}_{s}\right)+\frac{1}{2}\left(1-\frac{1}{\sigma}\right) \hat{C}_{s}^{2}\right]-Y v_{y}(\cdot)\left[\hat{Y}_{s}\left(1+\frac{h}{\psi} \hat{\xi}_{s}\right)\right. \\
& \left.+\frac{1}{2}\left(1+\frac{1}{\psi}\right) \hat{Y}_{s}^{2}+\frac{1}{2} \frac{(\psi+\epsilon) \epsilon \gamma}{\psi(1-\gamma \beta)(1-\gamma)} \pi_{H, t}^{2}\right]+\operatorname{tip}(3)
\end{aligned}
$$

Define 'natural' variables as values that would occur without the nominal inertia and international risk sharing distortions. All natural variables will be a function of $\hat{\xi}$ and $\hat{Y}^{*}$, world output disequilibrium. With price flexibility and a constant output subsidy, the mark-up over marginal costs will be constant, so from (22) we can write to first order

$$
0=\left[\hat{y}^{n} \frac{1}{\psi}+\hat{C}^{n} \frac{1}{\sigma}+\alpha \hat{S}^{n}+\hat{\xi}\left(\frac{h}{\psi}+\frac{g}{\sigma}\right)\right]
$$


An identical expression occurs in the closed economy, except that the term in $S$ obviously disappears.

In a closed economy, $\hat{C}=\hat{Y}$, so welfare can be further simplified as

$$
W_{s}^{\text {closed }}=U_{c}(\cdot) C\left[-\left(\frac{g}{\sigma}+\frac{h}{\psi}\right) \hat{C}_{s} \hat{\xi}_{s}-\frac{1}{2}\left(\frac{1}{\sigma}+\frac{1}{\psi}\right) \hat{C}_{s}^{2}-V_{z} \hat{y}(z) \frac{1}{2}\left(\frac{1}{\psi}+\frac{1}{\epsilon}\right)\right]+\operatorname{tip}(3)
$$

and so we can eliminate the terms in $\hat{\xi}$ to give

$$
\begin{aligned}
W_{s}^{\text {closed }} & =U_{c}(\cdot) C\left[\hat{C}_{s} \hat{C}_{s}^{n}\left(\frac{1}{\sigma}+\frac{1}{\psi}\right)-\frac{1}{2}\left(\frac{1}{\sigma}+\frac{1}{\psi}\right) \hat{C}_{s}^{2}-V_{z} \hat{y}_{s}(z) \frac{1}{2}\left(\frac{1}{\psi}+\frac{1}{\epsilon}\right)\right]+\operatorname{tip}(3) \\
& =U_{c}(\cdot) C\left[-\frac{1}{2}\left(\frac{1}{\sigma}+\frac{1}{\psi}\right)\left(\hat{C}_{s}-\hat{C}_{s}^{n}\right)^{2}-V_{z} \hat{y}_{s}(z) \frac{1}{2}\left(\frac{1}{\psi}+\frac{1}{\epsilon}\right)\right]+\operatorname{tip}(3)
\end{aligned}
$$

The second line notes the relationship between $\hat{C}^{2}$ and $\hat{C} \hat{C}^{n}$ to simplify in terms of the "consumption gap' $\hat{C}-\hat{C}^{n}$, bearing in mind that terms in $\hat{C}^{n}$ alone can be added to tip. Thus policy can increase welfare in two ways: by reducing the variance of output (across goods) and keeping output/consumption close to its natural level.

In an open economy we use the same procedure. Here, $\hat{C} \neq \hat{Y}$ so we cannot eliminate linear terms immediately, but instead we use second-order approximation to risk-sharing condition and aggregate demand.

A second order expansion of the aggregate demand curve was derived in (70)

$$
\begin{aligned}
\hat{Y} & =(1-\alpha) \hat{C}+\alpha \hat{C}^{*}+\alpha \eta(2-\alpha) \hat{S}+\frac{1}{2} \eta^{2} \alpha^{2}(2-\alpha) \hat{S}^{2} \\
& +\alpha \eta \hat{S} \hat{C}^{*}+\alpha \eta(1-\alpha) \hat{S} \hat{C}+\frac{1}{2}(1-\alpha) \hat{C}^{2}-\frac{1}{2} \hat{Y}^{2}+\frac{1}{2} \alpha \hat{C}^{* 2}
\end{aligned}
$$

while a second order expansion of the risk sharing condition (assuming $\hat{\xi}_{s}^{*}=\hat{\xi}_{s}$ ) implies

$$
\begin{aligned}
\hat{C} & =\sigma(1-\alpha) \hat{S}-\frac{1}{2} b \hat{C}^{2}+\frac{1}{2} \sigma \alpha(1-\alpha)(\eta-2) \hat{S}^{2} \\
& -d \hat{C} \hat{\xi}-(1-\alpha) \hat{C} \hat{S}-(1-\alpha) g \hat{S} \hat{\xi}+\hat{Y}_{s}^{*}-\sigma \hat{\zeta}-g\left(\hat{\xi}-\hat{\xi}^{*}\right)+\frac{1}{2} b \hat{C}^{* 2} \\
& -\frac{1}{2} g a \hat{\xi}^{2}-\frac{1}{2} \sigma \hat{\zeta}^{2}+\hat{C}^{*} \hat{\zeta}+g \hat{\xi}^{*} \hat{\zeta}+\frac{1}{2} g a \hat{\xi}^{* 2}+d \hat{C}^{*} \hat{\xi}^{*}
\end{aligned}
$$

and $\sigma$ and $g$ are defined in (73)-(74). These two equations can be solved for $\hat{C}$ and $\hat{S}$ :

$$
\begin{aligned}
\hat{C} & =\Phi \hat{Y}+(1-\Phi) \hat{Y}^{*}-\Phi \frac{\eta \alpha(2-\alpha)}{\sigma(1-\alpha)}\left(\sigma \hat{\zeta}+g\left(\hat{\xi}-\hat{\xi}^{*}\right)\right) \\
& +\frac{1}{2} \Phi \hat{Y}^{2}-\Phi \alpha^{2} \eta(2-\alpha) \hat{S}^{2}-\frac{1}{2} \frac{\Phi}{\Phi_{b}} \hat{C}^{2}-\Phi \eta \alpha \hat{S} \hat{Y}^{*} \\
& +\Phi \frac{\eta \alpha}{\sigma}(\sigma(1-\alpha)+(2-\alpha)) \hat{S} \hat{C}-\eta \alpha \Phi(2-\alpha)\left(d+\frac{g}{\sigma}\right) \hat{S} \hat{\xi}
\end{aligned}
$$




$$
\begin{aligned}
\hat{S} & =\frac{\Phi}{\sigma(1-\alpha)} Y+\frac{\Phi}{2 \sigma(1-\alpha)} \hat{Y}^{2}+\Phi\left(\hat{\zeta}+\frac{g}{\sigma}\left(\hat{\xi}-\hat{\xi}^{*}\right)\right)-\frac{\Phi}{\sigma(1-\alpha)} \hat{Y}^{*} \\
& -\frac{1}{2} \alpha \eta\left(1-\frac{2(1-\alpha)}{\eta} \Phi\right) \hat{S}^{2}+\frac{(b-1)}{2 \sigma} \Phi \hat{C}^{2} \\
& +\Phi \frac{(1-\alpha-\alpha \eta)}{\sigma} \hat{C} \hat{S}-\Phi \frac{\eta \alpha}{\sigma(1-\alpha)} \hat{S} \hat{Y}^{*}+\Phi(1-\alpha)\left(d+\frac{g}{\sigma}\right) \hat{S} \hat{\xi}
\end{aligned}
$$

where we used the first-order approximation

$$
\hat{C}=\sigma(1-\alpha) \hat{S}+\hat{Y}^{*}-\sigma \hat{\zeta}-g\left(\hat{\xi}-\hat{\xi}^{*}\right)
$$

and denoted

$$
\begin{aligned}
\Phi & =\frac{\sigma(1-\alpha)}{\left((2-\alpha) \eta \alpha+\sigma(1-\alpha)^{2}\right)} \\
\Phi_{b} & =\frac{\sigma(1-\alpha)}{\left(b(2-\alpha) \eta \alpha+\sigma(1-\alpha)^{2}\right)}
\end{aligned}
$$

For iso-elastic utility $\left(d+\frac{g}{\sigma}=0\right) \hat{S} \hat{\xi}$ disappears:

$$
\begin{aligned}
\hat{C} & =\Phi \hat{Y}+\frac{1}{2} \Phi \hat{Y}^{2}-\Phi \alpha^{2} \eta(2-\alpha) \hat{S}^{2}-\frac{1}{2} \frac{\Phi}{\Phi b} \hat{C}^{2} \\
& -\Phi \alpha \eta \hat{S} \hat{Y}^{*}-\Phi \frac{\alpha \eta}{\sigma}((2-\alpha)+\sigma(1-\alpha)) \hat{S} \hat{C}+t i p
\end{aligned}
$$

For future reference, the first order expansion is:

$$
\hat{C}=\Phi \hat{Y}+(1-\Phi) \hat{Y}^{*}-\Phi \frac{\eta \alpha(2-\alpha)}{\sigma(1-\alpha)}\left(\sigma \hat{\zeta}+g\left(\hat{\xi}-\hat{\xi}^{*}\right)\right)
$$

Substituting (101) into utility (assuming iso-elastic utility):

$$
\begin{aligned}
W_{s}=U_{c}(\cdot) C\left[\begin{array}{c}
\hat{C}_{s}\left(1-\frac{g}{\sigma} \hat{\xi}_{s}\right)+\frac{1}{2}\left(1-\frac{1}{\sigma}\right) \hat{C}_{s}^{2} \\
-\frac{Y v_{y}(\cdot)}{U_{c}(\cdot) C}\left(\hat{Y}_{s}\left(1+\frac{h}{\psi} \hat{\xi}_{s}\right)+\frac{1}{2}\left(1+\frac{1}{\psi}\right) \hat{Y}_{s}^{2}\right. \\
\left.+\frac{1}{2} \frac{(\psi+\epsilon) \epsilon \gamma}{\psi(1-\gamma \beta)(1-\gamma)} \pi_{H, s}^{2}\right)
\end{array}\right]+t i p(3) \\
=U_{c}(\cdot) C\left[\begin{array}{c}
\left(\Phi-\frac{1}{\mu}\right) \hat{Y}_{s}-\Phi \alpha^{2} \eta(2-\alpha) \hat{S}_{s}^{2}-\Phi \alpha \eta \hat{S}_{s} \hat{Y}_{s}^{*} \\
-\Phi \frac{\alpha \eta}{\sigma}((2-\alpha)+\sigma(1-\alpha)) \hat{S}_{s} \hat{C}_{s} \\
-\frac{1}{2}\left(\frac{\Phi}{\Phi_{b}}-\left(1-\frac{1}{\sigma}\right)\right) \hat{C}_{s}^{2} \\
+\left(\frac{1}{\sigma} \Phi+\frac{1}{\mu \psi}\right) \hat{Y}_{s} \hat{\xi}_{s}-\frac{1}{2}\left(\frac{1}{\mu}\left(1+\frac{1}{\psi}\right)-\Phi\right) \hat{Y}_{s}^{2} \\
-\frac{1}{2} \frac{(\psi+\epsilon) \epsilon \gamma}{\mu \psi(1-\gamma \beta)(1-\gamma)} \pi_{H, s}^{2}
\end{array}\right]+t i p(3)
\end{aligned}
$$

where

$$
\frac{Y v_{y}(Y, 1)}{C u_{C}(C, 1)}=\frac{1}{\mu}
$$

This formula contains a single linear term $\left(\Phi-\frac{1}{\mu}\right) \hat{Y}_{s}$. In what follows, we show how to bring this formula into a form that contains only quadratic terms. Two approaches have been 
advanced in the literature. The first adds a subsidy that should be paid to producers in order to remove monopolistic distortions. This we consider in Section B.1. Alternatively, we can use one of remaining equations, the Phillips curve, and try to express output as a function of other economic variables and substitute it in formula (103). This is discussed in Section B.2.

\section{B.1 Using subsidy to eliminate linear terms}

We can assume a subsidy that changes the markup $\mu$ such that $\frac{1}{\mu}=\Phi$. In this case

$$
W_{s}=U_{c}(\cdot) C\left[\begin{array}{c}
-\Phi \alpha^{2} \eta(2-\alpha) \hat{S}_{s}^{2}-\Phi \alpha \eta \hat{S}_{s} \hat{Y}_{s}^{*} \\
-\Phi \frac{\alpha \eta}{\sigma}((2-\alpha)+\sigma(1-\alpha)) \hat{S}_{s} \hat{C}_{s}-\frac{1}{2}\left(\frac{\Phi}{\Phi_{b}}-\left(1-\frac{1}{\sigma}\right)\right) \hat{C}_{s}^{2} \\
+\Phi\left(\frac{1}{\sigma}+\frac{1}{\psi}\right) \hat{Y}_{s} \hat{\xi}_{s}-\frac{1}{2} \Phi \frac{1}{\psi} \hat{Y}_{s}^{2}-\frac{1}{2} \frac{\Phi(\psi+\epsilon) \epsilon \gamma}{\psi(1-\gamma \beta)(1-\gamma)} \pi_{H, s}^{2}
\end{array}\right]+\operatorname{tip}(3)
$$

which is a quadratic form. Using the expression for $\hat{\xi}$ in terms of natural rates (92):

$$
\begin{aligned}
\hat{\xi} & =\frac{\psi \sigma}{\psi(\psi+\sigma)} \hat{Y}^{n}+\frac{\psi \sigma}{(\psi+\sigma) \sigma} \hat{C}^{n}+\frac{\alpha \psi \sigma}{(\psi+\sigma)} \hat{S}^{n} \\
& =\frac{(\sigma(1-\alpha)+\psi)}{(\psi+\sigma)} \hat{C}^{n}+\frac{\sigma}{(\psi+\sigma)} \alpha \hat{Y}^{* n}+\sigma \alpha \frac{(\eta(2-\alpha)+\psi)}{(\psi+\sigma)} \hat{S}^{n}
\end{aligned}
$$

allows us to replace the quadratic terms in the consumption gap in the welfare function:

$$
W_{s}=-U_{c}(\cdot) C \Phi\left[\begin{array}{c}
\alpha^{2} \eta(2-\alpha)\left(\hat{S}_{s}-\hat{S}_{s}^{n}\right)^{2}+\frac{1}{2}\left(\frac{(1-\sigma)}{\sigma \Phi}+\frac{1}{\Phi_{b}}\right)\left(\hat{C}_{s}-\hat{C}_{s}^{n}\right)^{2}+\frac{1}{2} \frac{1}{\psi}\left(\hat{Y}_{s}-\hat{Y}_{s}^{n}\right) \\
+\frac{\alpha \eta}{\sigma}((2-\alpha)+\sigma(1-\alpha))\left(\hat{S}_{s}-\hat{S}_{s}^{n}\right)\left(\hat{C}_{s}-\hat{C}_{s}^{n}\right) \\
-\alpha \hat{C}_{s}\left[\frac{(1+\sigma)(2-\alpha) \eta}{\sigma^{2}(1-\alpha)} \hat{C}_{s}^{n}+\frac{[\varpi-\eta] \sigma(1-\alpha)^{2}-[1-(\varpi+1) \alpha] \eta(2-\alpha)}{\sigma(1-\alpha)} \hat{S}_{s}^{n}\right] \\
+\alpha \eta\left[2 \alpha(2-\alpha) \hat{S}_{s}^{n}+\frac{((2-\alpha)+\sigma(1-\alpha))}{\sigma} \hat{C}_{s}^{n}+\hat{Y}_{s}^{*}\right] \hat{S}_{s} \\
+\frac{1}{2} \frac{(\psi+\epsilon) \epsilon \gamma}{\psi(1-\gamma \beta)(1-\gamma)} \pi_{H, s}^{2}
\end{array}\right]+\operatorname{tip}(3)
$$

From the linear approximation to aggregate demand (71) we express $\hat{C}$ as function of $\hat{Y}, \hat{Y}^{*}$ and $\hat{S}$, substitute it into (104) and rewrite it as:

$$
W_{s}=U_{c}(\cdot) C \Phi\left[\begin{array}{c}
\frac{1}{2} \frac{\left((1-\alpha)^{2}(\eta(2-\alpha)+2 \sigma(1-\alpha)(\eta-1))+\alpha \eta^{2}(2-\alpha)^{2}\right)(2-\alpha) \eta \alpha^{2}}{(1-\alpha)^{3} \sigma} \hat{S}_{s}^{2} \\
-\left(\sigma(1-\alpha)^{3}+\alpha \eta(2-\alpha)^{2}\right) \frac{\eta \alpha}{(1-\alpha)^{3} \sigma} \hat{S}_{s} \hat{Y}_{s} \\
+\left(-\sigma(1-\alpha)^{4}+\alpha^{2} \eta(2-\alpha)^{2}\right) \frac{\eta \alpha}{(1-\alpha)^{3} \sigma} \hat{S}_{s} \hat{Y}_{s}^{*} \\
-\frac{1}{2}\left(\frac{1}{\psi}+\frac{\left((1-\alpha)^{2}-\eta \alpha(2-\alpha)\right)}{\sigma(1-\alpha)^{3}}\right) \hat{Y}_{s}^{2} \\
+\left(\frac{1}{\sigma}+\frac{1}{\psi}\right) \hat{Y}_{s} \hat{\xi}_{s}+\frac{1}{2} \frac{\left((1-\alpha)^{2}-\eta \alpha(2-\alpha)\right)}{\sigma(1-\alpha)^{3}} 2 \alpha \hat{Y}_{s} \hat{Y}_{s}^{*} \\
-\frac{1}{2} \frac{(\psi+\epsilon) \epsilon \gamma}{\psi(1-\gamma \beta)(1-\gamma)} \pi_{H, s}^{2}
\end{array}\right]+\operatorname{tip}(3)
$$

using the expression for $\hat{\xi}$ in terms of natural rates again, we get:

$$
\begin{aligned}
W_{s} & =U_{c}(\cdot) C\left\{A_{1}\left(\hat{Y}_{s}-\hat{Y}_{s}^{n}\right)^{2}+A_{2}\left(\hat{S}_{s}-\hat{S}_{s}^{n}\right)^{2}+A_{3}\left(\hat{S}_{s}-\hat{S}_{s}^{n}\right)\left(\hat{Y}_{s}-\hat{Y}_{s}^{n}\right)\right. \\
& +\hat{S}_{s}\left(A_{41} \hat{Y}_{s}^{n}+A_{42} \hat{S}_{s}^{n}+A_{45} \hat{Y}_{s}^{*}\right) \\
& \left.+\hat{Y}_{s}\left(A_{51} \hat{Y}_{s}^{n}+A_{52} \hat{S}_{s}^{n}+A_{55} \hat{Y}_{s}^{*}\right)+A_{6} \pi_{H, s}^{2}\right\}+\operatorname{tip}(3)
\end{aligned}
$$


where

$$
\begin{aligned}
& A_{1}=-\frac{1}{2} \Phi\left(\frac{1}{\psi}+\frac{\left((1-\alpha)^{2}-\eta \alpha(2-\alpha)\right)}{\sigma(1-\alpha)^{3}}\right) \\
& A_{2}=-\frac{1}{2} \frac{\Phi \alpha^{2}}{(1-\alpha)^{3} \sigma}(2-\alpha) \eta\left((1-\alpha)^{2} 2 \sigma(1-\alpha)(1-\eta)-(2-\alpha) \eta\left((1-\alpha)^{2}+\alpha \eta(2-\alpha)\right)\right) \\
& A_{3}=-\Phi \frac{\eta \alpha\left(\sigma(1-\alpha)^{3}+\alpha \eta(2-\alpha)^{2}\right)}{(1-\alpha)^{3} \sigma}, \quad A_{41}=-\Phi \frac{\eta \alpha\left(\sigma(1-\alpha)^{3}+\alpha \eta(2-\alpha)^{2}\right)}{(1-\alpha)^{3} \sigma} \\
& A_{42}=\frac{\Phi}{(1-\alpha)^{3} \sigma}(2-\alpha) \eta \alpha^{2}\left(\alpha \eta^{2}(2-\alpha)^{2}+(1-\alpha)^{2}(\eta(2-\alpha)+2 \sigma(1-\alpha)(\eta-1))\right) \\
& A_{45}=\Phi \frac{\eta \alpha\left(\alpha^{2} \eta(2-\alpha)^{2}-\sigma(1-\alpha)^{4}\right)}{(1-\alpha)^{3} \sigma}, \quad A_{51}=\Phi \frac{\eta \alpha(2-\alpha)}{\sigma(1-\alpha)^{3}} \\
& A_{52}=-\frac{\Phi \alpha}{(1-\alpha)^{3} \sigma}\left(\alpha \eta^{2}(2-\alpha)^{2}-(\sigma(1-\eta)(1-\alpha)-\eta(2-\alpha))(1-\alpha)^{2}\right) \\
& A_{55}=-\Phi \frac{\alpha^{2} \eta(2-\alpha)}{\sigma(1-\alpha)^{3}}, \quad A_{6}=-\frac{1}{2} \Phi \frac{(\psi+\epsilon) \epsilon \gamma}{\psi(1-\gamma \beta)(1-\gamma)}
\end{aligned}
$$

\section{B.2 Using Phillips Curve to eliminate linear terms}

In this Section's derivations we closely follow Benigno and Woodford (2004). Our notation is made as close to theirs as possible. We make an assumption that our utility is iso-elastic, as this substantially simplifies derivations.

As discussed above, our task is to eliminate the remaining linear terms in (103) without using a subsidy to producers. Instead, it was suggested by Sutherland (2002b) and illustrated by Benigno and Woodford (2004), to use one of remaining equations, Phillips curve. However, the Phillips curve is a dynamic relationship, and by using it, we eliminate unnecessary linear terms from the utility representation, but introduce dynamic structure.

We have previously derived (81) which depends on $z_{t}+\frac{1}{2} z_{t} X_{t}$. We now derive $z_{t}+\frac{1}{2} z_{t} X_{t}$ in terms of conventional variables. To do so we use second-order approximations to the risk sharing equation and aggregate demand (70) and (66). These relationships can be solved to yield the term of trade $\hat{S}$ and consumption $\hat{C}$ (98) and (97). Using the linear approximation: $\hat{C}=\left(\frac{1}{(1-\alpha)} \hat{Y}-\frac{\alpha}{(1-\alpha)} \hat{Y}^{*}-\frac{\alpha \eta(2-\alpha)}{(1-\alpha)} \hat{S}\right)$ we can get rid out of $\hat{C}$ in the RHS of both (97) and (98). The obtained expressions will contain two terms, involving $\hat{\xi}: \hat{S} \hat{\xi}$ and $\hat{Y} \hat{\xi}$. We can use $\hat{S}=\left(\hat{Y}-\hat{Y}^{*}+(1-\alpha)\left(\sigma \hat{\zeta}+g\left(\hat{\xi}-\hat{\xi}^{*}\right)\right)\right) /\left(\alpha \eta(2-\alpha)+\sigma(1-\alpha)^{2}\right)$ to substitute into $\hat{S} \hat{\xi}$ in order to have only one term with $\hat{\xi}: \hat{Y} \hat{\xi}$. By assuming the iso-elastic version we eliminate it as well and end up with ${ }^{10}$ :

\footnotetext{
${ }^{10}$ For an iso-elastic function, equality $(75)$ is used.
} 


$$
\begin{aligned}
\hat{C} & =c_{Y} \hat{Y}+c_{X} \hat{Y}^{*}+c_{Z}\left(\sigma \hat{\zeta}+g\left(\hat{\xi}-\hat{\xi}^{*}\right)\right) \\
& +c_{Y Y} \hat{Y}^{2}+c_{S S} \hat{S}^{2}+c_{X Y} \hat{Y}^{*} \hat{Y}+c_{S Y} \hat{S} \hat{Y}+c_{S X} \hat{S} \hat{Y}^{*} \\
\hat{S} & =s_{Y} \hat{Y}+s_{X} \hat{Y}^{*}+s_{Z}\left(\sigma \hat{\zeta}+g\left(\hat{\xi}-\hat{\xi}^{*}\right)\right) \\
& +s_{Y Y} \hat{Y}^{2}+s_{S S} \hat{S}^{2}+s_{X Y} \hat{Y}^{*} \hat{Y}+s_{S Y} \hat{S} \hat{Y}+s_{S X} \hat{S} \hat{Y}^{*}
\end{aligned}
$$

where:

$$
\begin{aligned}
& c_{Y}=\Phi, \quad c_{X}=(1-\Phi), \quad c_{Z}=-\Phi \frac{\eta \alpha(2-\alpha)}{\sigma(1-\alpha)}, \\
& c_{Y Y}=\frac{1}{2} \Phi \frac{\left(\eta(2-\alpha)-\sigma^{2}(1-\alpha)^{2}\right) \alpha}{(1-\alpha)^{3} \sigma^{2}}, \\
& c_{S S}=-\frac{1}{2} \Phi \frac{(\alpha-2) \eta \alpha^{2}}{(1-\alpha)^{3} \sigma^{2}}\left(-\sigma \eta(1-\alpha)^{2}(2(2-\alpha)+\sigma(4-3 \alpha))\right. \\
& \left.-2 \sigma^{2}(1-\alpha)^{3}+\alpha \eta^{2}(2-\alpha)^{2}\right), \\
& c_{X Y}=\Phi \frac{\left(\sigma^{2}(1-\alpha)^{2}-(2-\alpha) \eta \alpha\right) \alpha}{\sigma^{2}(1-\alpha)^{3}} \\
& c_{S Y}=-\frac{\Phi \eta \alpha}{(1-\alpha)^{3} \sigma^{2}}\left(\alpha \eta(\alpha-2)^{2}\right. \\
& \left.-\sigma(2-\alpha)(1-\alpha)^{2}-\sigma^{2}(3-2 \alpha)(1-\alpha)^{2}\right), \\
& c_{S X}=\frac{\Phi \eta \alpha}{(1-\alpha)^{3} \sigma^{2}}\left(\alpha^{2} \eta(2-\alpha)^{2}\right. \\
& \left.-\sigma \alpha(2-\alpha)(1-\alpha)^{2}-\sigma^{2}\left(1+2 \alpha-2 \alpha^{2}\right)(1-\alpha)^{2}\right) . \\
& s_{Y}=\frac{\Phi}{\sigma(1-\alpha)}, \quad s_{X}=-\frac{\Phi}{\sigma(1-\alpha)}, \quad s_{Z}=\Phi, \quad s_{Y Y}=-\Phi \frac{1}{2} \frac{(\alpha \sigma+1)}{(1-\alpha)^{2} \sigma^{2}} \\
& s_{S S}=-\frac{1}{2} \frac{\Phi \alpha}{(1-\alpha)^{2} \sigma^{2}}\left(-\sigma \eta(1-\alpha)^{2}(2(\alpha-2)-\sigma(1-\alpha))\right. \\
& \left.-2 \sigma^{2}(1-\alpha)^{3}+\alpha \eta^{2}(2-\alpha)(\sigma+2-\alpha)\right) \\
& s_{X Y}=\Phi \frac{(1+\sigma)}{\sigma^{2}(1-\alpha)^{2}} \alpha, \quad s_{S Y}=\Phi \frac{\left(\sigma(1-\alpha)^{2}+\alpha \eta(\sigma-\alpha+2)\right)}{(1-\alpha)^{2} \sigma^{2}} \\
& s_{S X}=-\Phi \frac{\left(\sigma(1-\alpha)^{2}+\eta\left(2 \alpha+\sigma-\alpha^{2}\right)\right) \alpha}{(1-\alpha)^{2} \sigma^{2}}
\end{aligned}
$$

We now can substitute (106) and (107) into $z+\frac{1}{2} z X$ to obtain: 


$$
\begin{aligned}
z+\frac{1}{2} z X & =\left(\frac{1}{\psi} \hat{Y}+\frac{1}{\sigma} \hat{C}-\left(1-\frac{1}{\sigma}+\frac{1}{\psi}\right) \hat{\xi}+\alpha \hat{S}\right)\left(\left(\frac{1}{\psi}+2\right) \hat{Y}\right. \\
& \left.-\frac{1}{\sigma} \hat{C}+\left(\frac{1}{\sigma}-\frac{1}{\psi}\right) \hat{\xi}-\alpha \hat{S}\right)+\alpha \hat{S}+\frac{1}{\psi} \hat{Y}+\frac{1}{\sigma} \hat{C} \\
& -\left(\frac{1}{\sigma}+\frac{1}{\psi}\right) \hat{\xi}-\frac{1}{2} \alpha(\eta-1)(1-\alpha) \hat{S}^{2} \\
& =\lambda_{Y} \hat{Y}+\lambda_{X Y} \hat{Y} \hat{Y}^{*}+\lambda_{Y Y} \hat{Y}^{2}+\lambda_{S Y} \hat{S} \hat{Y}+\lambda_{S X} \hat{S} \hat{Y}^{*}+\lambda_{S S} \hat{S}^{2}+\lambda_{Y \xi} \hat{Y} \hat{\xi}
\end{aligned}
$$

where

$$
\begin{aligned}
\lambda_{Y} & =\alpha s_{Y}+\frac{1}{\sigma} c_{Y}+\frac{1}{\psi} \\
\lambda_{X Y} & =\alpha s_{X Y}+\frac{1}{\sigma} c_{X Y}-2 \frac{\alpha}{\sigma(1-\alpha)}+2 \frac{\alpha}{\sigma^{2}(1-\alpha)^{2}} \\
\lambda_{Y Y} & =\frac{2}{\psi}+\frac{1}{\psi^{2}}+\frac{2}{\sigma(1-\alpha)}-\frac{1}{\sigma^{2}(1-\alpha)^{2}}+\alpha s_{Y^{2}}+\frac{1}{\sigma} c_{Y^{2}} \\
\lambda_{S Y} & =-2 \frac{((1-\alpha) \sigma-\eta(2-\alpha))(\alpha \sigma-\sigma+1) \alpha}{(\alpha-1)^{2} \sigma^{2}}+\alpha s_{S Y}+\frac{1}{\sigma} c_{S Y} \\
\lambda_{S X} & =\alpha s_{S X}+\frac{1}{\sigma} c_{S X}+2 \frac{\alpha^{2}}{\sigma(1-\alpha)} \\
& -4 \eta \frac{\alpha^{2}}{\sigma^{2}(1-\alpha)^{2}}+2 \eta \frac{\alpha^{3}}{\sigma^{2}(1-\alpha)^{2}} \\
\lambda_{S S} & =-\frac{1}{2} \frac{\alpha}{(\alpha-1)^{2} \sigma^{2}}\left(-\sigma \eta(\alpha-1)\left(\sigma(\alpha-1)^{2}-4 \alpha(2-\alpha)\right)\right. \\
& \left.+\sigma^{2}(3 \alpha-1)(\alpha-1)^{2}+2 \alpha \eta^{2}(\alpha-2)^{2}\right) \\
& +\alpha s_{S^{2}}+\frac{1}{\sigma} c_{S^{2}} \\
\lambda_{Y \xi} & =\frac{2}{\sigma}+\frac{2}{\sigma \psi}-\frac{3}{\psi}-2-\frac{2}{\psi^{2}}+\frac{1}{\eta \alpha(2-\alpha)+\sigma(1-\alpha)^{2}}
\end{aligned}
$$

We have derived the general form for second-order approximation of the welfare function (91), so substituting $\hat{C}_{t}$ from (106) into the part of (91) which depends on consumption, we obtain for the iso-elastic version

$$
\hat{C}-\frac{g}{\sigma} \hat{C} \hat{\xi}+\frac{1}{2}\left(1-\frac{1}{\sigma}\right) \hat{C}^{2}=\mu_{Y} \hat{Y}+\mu_{X Y} \hat{Y} \hat{Y}^{*}+\mu_{Y Y} \hat{Y}^{2}+\mu_{S Y} \hat{S} \hat{Y}+\mu_{S X} \hat{S} \hat{Y}^{*}+\mu_{S S} \hat{S}^{2}+\mu_{Y \xi} \hat{Y} \hat{\xi}
$$

where

$$
\begin{aligned}
& \mu_{Y}=c_{Y}, \quad \mu_{X Y}=c_{X Y}+\frac{\alpha(1-\sigma)}{\sigma(1-\alpha)^{2}}, \quad \mu_{Y Y}=c_{Y^{2}}-\frac{(1-\sigma)}{2 \sigma(1-\alpha)^{2}}, \\
& \mu_{S Y}=c_{S Y}+\eta \frac{\alpha(1-\sigma)(2-\alpha)}{\sigma(1-\alpha)^{2}}, \quad \mu_{S X}=c_{S X}-\eta \frac{\alpha^{2}(2-\alpha)(1-\sigma)}{\sigma(1-\alpha)^{2}}, \\
& \mu_{S S}=c_{S^{2}}-\frac{1}{2} \eta^{2} \frac{\alpha^{2}(2-\alpha)^{2}(1-\sigma)}{\sigma(1-\alpha)^{2}}, \quad \mu_{Y \xi}=\frac{1}{\sigma} c_{Y} .
\end{aligned}
$$


We can write that

$$
\begin{aligned}
\mathcal{W}_{t_{0}} & =\sum_{t=t_{0}}^{\infty} \beta^{t-t_{0}}\left\{C u_{C}(C, 1)\left[\hat{C}_{t}-\frac{g}{\sigma} \hat{C}_{t} \hat{\xi}_{t}+\frac{1}{2}\left(1-\frac{1}{\sigma}\right) \hat{C}_{t}^{2}\right]\right. \\
& \left.-Y v_{y}(Y, 1)\left[\hat{Y}_{t}+\frac{h}{\psi} \hat{Y}_{t} \hat{\xi}_{t}+\frac{1}{2}\left(1+\frac{1}{\psi}\right) \hat{Y}_{t}^{2}+\frac{1}{2}\left(\frac{1}{\psi}+\frac{1}{\epsilon}\right) v a r_{z} \hat{y}_{t}(z)\right]\right\} \\
& =C u_{C}(C, 1) \sum_{t=t_{0}}^{\infty} \beta^{t-t_{0}}\left\{\left(\mu_{Y}-\frac{1}{\mu}\right) \hat{Y}_{t}+\mu_{X Y} \hat{Y}_{t} \hat{Y}_{t}^{*}\right. \\
& +\left(\mu_{Y Y}-\frac{1}{2} \frac{1}{\mu}\left(1+\frac{1}{\psi}\right)\right) \hat{Y}_{t}^{2}+\mu_{S Y} \hat{S}_{t} \hat{Y}_{t}+\mu_{S X} \hat{S}_{t} \hat{Y}_{t}^{*}+\mu_{S S} \hat{S}_{t}^{2} \\
& \left.+\left(\mu_{Y \xi}-\frac{h}{\mu \psi}\right) \hat{Y}_{t} \hat{\xi}_{t}-\frac{1}{2}\left(\frac{1}{\psi}+\frac{1}{\epsilon}\right) \frac{\gamma \epsilon^{2}}{\mu(1-\gamma \beta)(1-\gamma)} \pi_{H, t}^{2}\right\}
\end{aligned}
$$

where

$$
\frac{Y v_{y}(Y, 1)}{C u_{C}(C, 1)}=\frac{1}{\mu}=-\frac{1-\epsilon}{\epsilon}
$$

as no subsidy is imposed, and

$$
\begin{aligned}
V_{t_{0}} & =\sum_{t=t_{0}}^{\infty} \beta^{t-t_{0}} \kappa\left(z_{t}+\frac{1}{2} z_{t} X_{t}\right)+\frac{1}{2} \frac{(1-\epsilon)-\gamma \epsilon\left(\frac{1}{\psi}+1\right)}{(1-\gamma)} \sum_{t=t_{0}}^{\infty} \beta^{t-t_{0}} \pi_{H, t}^{2} \\
& =\sum_{t=t_{0}}^{\infty} \beta^{t-t_{0}} \kappa\left(\lambda_{Y} \hat{Y}_{t}+\lambda_{X Y} \hat{Y}_{t} \hat{Y}_{t}^{*}+\lambda_{Y Y} \hat{Y}_{t}^{2}+\lambda_{S Y} \hat{S}_{t} \hat{Y}_{t}+\lambda_{S X} \hat{S}_{t} \hat{Y}_{t}^{*}+\lambda_{S S} \hat{S}_{t}^{2}+\lambda_{Y \xi} \hat{Y}_{t} \hat{\xi}_{t}\right) \\
& -\frac{1}{2} \epsilon \frac{\left(1-\frac{1}{\epsilon}\right)+\gamma\left(1+\frac{1}{\psi}\right)}{(1-\gamma)} \sum_{t=t_{0}}^{\infty} \beta^{t-t_{0}} \pi_{H, t}^{2}
\end{aligned}
$$

Now, we can eliminate linear term in $Y_{t}$ from $\mathcal{W}_{t_{0}}$ using expression for $V_{t_{0}}$ :

$$
\begin{aligned}
\mathcal{W}_{t_{0}} & =\theta V_{t_{0}}-Y v_{y}(Y, 1) \sum_{t=t_{0}}^{\infty} \beta^{t-t_{0}}\left\{\mu\left(\left(\mu_{Y}-\frac{1}{\mu}\right) \frac{\lambda_{X Y}}{\lambda_{Y}}-\mu_{X Y}\right) \hat{Y}_{t}^{*} Y_{t}\right. \\
& +\mu\left(\frac{1}{2} \frac{1}{\mu}\left(1+\frac{1}{\psi}\right)+\left(\mu_{Y}-\frac{1}{\mu}\right) \frac{\lambda_{Y Y}}{\lambda_{Y}}-\mu_{Y Y}\right) Y_{t}^{2} \\
& +\mu\left(\left(\mu_{Y}-\frac{1}{\mu}\right) \frac{\lambda_{S Y}}{\lambda_{Y}}-\mu_{S Y}\right) S_{t} Y_{t}+\mu\left(\left(\mu_{Y}-\frac{1}{\mu}\right) \frac{\lambda_{S X}}{\lambda_{Y}}-\mu_{S X}\right) S_{t} \hat{Y}_{t}^{*} \\
& +\mu\left(\left(\mu_{Y}-\frac{1}{\mu}\right) \frac{\lambda_{S S}}{\lambda_{Y}}-\mu_{S S}\right) S_{t}^{2} \\
& +\mu\left(\left(\mu_{Y}-\frac{1}{\mu}\right) \frac{\lambda_{Y \xi}}{\lambda_{Y}}-\left(\mu_{Y \xi}-\frac{1}{\mu} \frac{h}{\psi}\right)\right) Y_{t} \xi_{t} \\
& \left.+\frac{1}{2} \frac{\epsilon}{(1-\gamma)}\left(\left(\frac{1}{\psi}+\frac{1}{\epsilon}\right) \frac{\gamma \epsilon}{(1-\gamma \beta)}-\mu \frac{\left(\mu_{Y}-r\right)}{\lambda_{Y}} \frac{\left(1-\frac{1}{\epsilon}\right)+\gamma\left(1+\frac{1}{\psi}\right)}{\kappa}\right) \pi_{H, t}^{2}\right\} \\
& =\theta V_{t_{0}}-Y v_{y} \sum_{t=t_{0}}^{\infty} \beta^{t-t_{0}}\left\{\nu_{X Y} Y_{t} \hat{Y}_{t}^{*}+\nu_{Y Y} Y_{t}^{2}+\nu_{S Y} S_{t} Y_{t}\right. \\
& \left.+\nu_{S X} S_{t} \hat{Y}_{t}^{*}+\nu_{S S} S_{t}^{2}+\nu_{Y \xi} Y_{t} \xi_{t}+\nu_{\pi \pi} \pi_{H, t}^{2}\right\}
\end{aligned}
$$


where

$$
\theta=\frac{C u_{C}(C, 1)\left(\mu_{Y}-\frac{1}{\mu}\right)}{\kappa \lambda_{Y}}
$$

We now try to write the formula in gap variables. As shock $\hat{\xi}$ enters in quadratic terms only, it is enough to use linear approximation to equilibrium condition in order to define natural rates, see formula (105) and

$$
\hat{\xi}=\frac{(\psi+\sigma(1-\alpha))}{(\psi+\sigma)(1-\alpha)} \hat{Y}^{n}-\frac{\psi \alpha \sigma}{\psi+\sigma}\left(\frac{\eta(2-\alpha)}{\sigma(1-\alpha)}-1\right) \hat{S}^{n}-\frac{\psi \alpha}{(\psi+\sigma)(1-\alpha)} \hat{Y}^{* n}
$$

Therefore:

$$
\begin{aligned}
\mathcal{W}_{t_{0}} & =\theta V_{t_{0}}-Y v_{y} \sum_{t=t_{0}}^{\infty} \beta^{t-t_{0}}\left\{\nu_{Y Y}\left(\hat{Y}_{t}-\hat{Y}_{t}^{n}\right)^{2}+\nu_{S S}\left(\hat{S}_{t}-\hat{S}_{t}^{n}\right)^{2}+\nu_{S Y}\left(\hat{S}_{t}-\hat{S}_{t}^{n}\right)\left(\hat{Y}_{t}-\hat{Y}_{t}^{n}\right)\right. \\
& +\hat{Y}_{t}\left(\left(\nu_{S Y}-\frac{\psi \alpha \sigma(\eta(2-\alpha)-\sigma(1-\alpha))}{(\psi+\sigma) \sigma(1-\alpha)} \nu_{Y \xi}\right) \hat{S}_{t}^{n}+\left(2 \nu_{Y Y}+\frac{(\psi+\sigma(1-\alpha))}{(\psi+\sigma)(1-\alpha)} \nu_{Y \xi}\right) \hat{Y}^{n}\right. \\
& \left.\left.+\left(\nu_{X Y}-\frac{\psi \alpha}{(\psi+\sigma)(1-\alpha)} \nu_{Y \xi}\right) \hat{Y}^{* n}\right)+\hat{S}_{t}\left(2 \nu_{S S} \hat{S}_{t}^{n}+\nu_{S Y} \hat{Y}_{t}^{n}+\nu_{S X} \hat{Y}_{t}^{* n}\right)+\nu_{\pi \pi} \pi_{H, t}^{2}\right\} \\
& =\theta V_{t_{0}}-Y v_{y} W+t i p(3)
\end{aligned}
$$

where

$$
\begin{aligned}
W & =A_{1}\left(\hat{Y}-\hat{Y}^{n}\right)^{2}+A_{2}\left(\hat{S}-\hat{S}^{n}\right)^{2}+A_{3}\left(\hat{S}-\hat{S}^{n}\right)\left(\hat{Y}-\hat{Y}^{n}\right) \\
& +\hat{S}\left(A_{41} \hat{S}^{n}+A_{42} \hat{Y}^{n}+A_{43} \hat{Y}^{* n}\right) \\
& +\hat{Y}\left(A_{51} \hat{S}^{n}+A_{52} \hat{Y}^{n}+A_{53} \hat{Y}^{* n}\right)+B_{6} \pi_{H}^{2}
\end{aligned}
$$

and

$$
\begin{aligned}
A_{1} & =\nu_{Y Y}, \quad A_{2}=\nu_{S S}, \quad A_{3}=\nu_{S Y}, \quad A_{41}=2 \nu_{S S}, \quad A_{43}=\nu_{S Y}, \quad A_{44}=\nu_{S X}, \\
A_{51} & =\nu_{S Y}-\frac{\psi \alpha \sigma(\eta(2-\alpha)-\sigma(1-\alpha))}{(\psi+\sigma) \sigma(1-\alpha)} \nu_{Y \xi}, \quad A_{52}=2 \nu_{Y Y}+\frac{(\psi+\sigma(1-\alpha))}{(\psi+\sigma)(1-\alpha)} \nu_{Y \xi}, \\
A_{53} & =\nu_{X Y}-\frac{\psi \alpha}{(\psi+\sigma)(1-\alpha)} \nu_{Y \xi}, \quad A_{6}=\nu_{\pi \pi} .
\end{aligned}
$$

